\title{
Experimental investigation of short scalelength density fluctuations in laser-produced plasmas
}

\author{
J.D. Moody, B.J. MacGowan, S.H. Glenzer, \\ R.K. Kirkwood, W.L. Kruer, D.S. Montgomery, \\ A.J. Schmitt, E.A. Williams, G.F. Stone
}

This article was submitted to the $41^{\text {st }}$ Annual Meeting of the Division of Plasma Physics, Seattle, WA, November 15-19, 1999

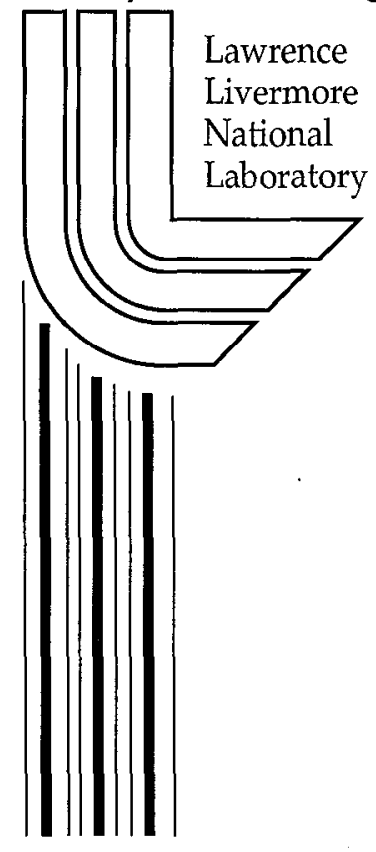

November 1, 1999 



\section{DISCLAIMER}

This document was prepared as an account of work sponsored by an agency of the United States Government. Neither the United States Government nor the University of California nor any of their employees, makes any warranty, express or implied, or assumes any legal liability or responsibility for the accuracy, completeness, or usefulness of any information, apparatus, product, or process disclosed, or represents that its use would not infringe privately owned rights. Reference herein to any specific commercial product, process, or service by trade name, trademark, manufacturer, or otherwise, does not necessarily constitute or imply its endorsement, recommendation, or favoring by the United States Government or the University of California. The views and opinions of authors expressed herein do not necessarily state or reflect those of the United States Government or the University of California, and shall not be used for advertising or product endorsement purposes.

This is a preprint of a paper intended for publication in a journal or proceedings. Since changes may be made before publication, this preprint is made available with the understanding that it will not be cited or reproduced without the permission of the author.

This report has been reproduced directly from the best available copy.

Available to DOE and DOE contractors from the

Office of Scientific and Technical Information

P.O. Box 62, Oak Ridge, TN 37831

Prices available from (423) 576-8401

http:/ / apollo.osti.gov/bridge/

Available to the public from the National Technical Information Service

U.S. Department of Commerce 5285 Port Royal Rd., Springfield, VA 22161

http://www.ntis.gov/

OR

Lawrence Livermore National Laboratory Technical Information Department's Digital Library http://www.llnl.gov/tid/Library.html 



\title{
Experimental investigation of short scalelength density fluctuations in laser-produced plasmas
}

\author{
J. D. Moody, B. J. MacGowan, S. H. Glenzer, R. K. Kirkwood, \\ W. L. Kruer, D. S. Montgomery ${ }^{1}$, A. J. Schmitt ${ }^{2}$, E. A. Williams, and G. F. Stone \\ Lawrence Livermore National Laboratory \\ University of California \\ Livermore, CA 94550 \\ and \\ ${ }^{1}$ Los Alamos National Laboratory \\ Los Alamos, NM \\ and \\ ${ }^{2}$ Naval Research Laboratory \\ Washington, $D C$
}

\begin{abstract}
The technique of near forward laser scattering is used to infer characteristics of intrinsic and controlled density fluctuations in laser-produced plasmas. Intrinsic fluctuations are studied in long scalelength plasmas where the fluctuations exhibit scalesizes related to the size of the intensity variations in the plasma forming and interaction beams. Stimulated Brillouin forward scattering and filamentation appear to be the primary mechanism through which these fluctuations originate. The beam spray which results from these fluctuations is important to understand since it can affect symmetry in an inertial confinement fusion (ICF) experiment. Controlled fluctuations are studied in foam and exploding foil targets. Forward scattered light from foam targets shows evidence that the initial target inhomogeneities remain after the target is laser heated. Forward scattered light from an exploding foil plasma shows that a regular intensity pattern can be used to produce a spatially correllated density fluctuation pattern. These results provide data which can be used to benchmark numerical models of beam spray.
\end{abstract}




\section{Introduction}

One aspect to achieving a complete physical description of laser-produced plasmas is understanding the role of low frequency density fluctuations. These fluctuations in a laser-plasma set the starting noise level for growth of instabilites such as stimulated Brillouin scattering (SBS) and filamentation. Continued growth of these instabilites beyond the noise level to saturation may be further affected by the fluctuations[1-3]. Low frequency fluctuations also affect laser propagation. For example, the laser light can undergo beam spray and increase its bandwidth as a result of active or passive scattering off the fluctuations. Fluctuations may arise from the initial structure of the target material, such as in the casc of a foam, or may be imposed on the target plasma by the ponderomotive/thermal pressure arising from the intensity structure within the laser beams. A comprehensive description of a laser-plasma includes quantification of the fluctuations and an understanding of how the fluctuations affect the laser-plasma interactions and laser propagation.

In addition to the basic physics aspects of fluctuations, the study of fluctuations is important for inertial confinement fusion (ICF) [ 4,5]. The current schemes for ICF ignition require a highly symmetric illumination of the fuel capsule by $\mathrm{x}$-rays (indirect drive) or laser light (direct drive). A concern for ICF is that fluctuations or laser-plasma instabilities will increase the angular spread (beam spray) of a beam which can cause illumination asymmetry [6,7] and substantially reduce the yield of an ICF target. Detailed measurements of forward scattered light in ignition relevant plasmas provide an experimental database for evaluating the severity of beam spray and for determining the physical processes involved in creating the beam spray. Forward scattered light experiments also help identify methods for reducing and controling the beam spray and testing the codes which model forward scattering:

We have conducted a series of experiments which use the technique of forward scattered light to investigate the character of intrinsic and controlled fluctuations. 
The distinction between these two types of fluctuations has to do with the process by which the fluctuations are made. Intrinsic fluctuations are not created intentionally but result in a plasma due to the complex interaction between the hydrodynamic evolution of the electron and ion densities and the ponderomotive/thermal pressure from the laser beams incident on the plasma. Experiments studying intrinsic fluctuations are done in a long scalelength plasma. The aim of these experiments is to determine the beam-spray and fluctuations which are characteristic of a plasma having a similar scalelength and density as the plasma in the central region of a NIF hohlraum while illuminated with a laser beam having similar intensity, $\mathrm{f} /$ number, and beam smoothing as a NIF laser beam. Forward scattered light measurements show small-angle and large-angle beam spreading. Interpretation of these measurements indicates that the small-angle beam spreading may arise from density inhomogeneities produced by intensity structure in the plasma forming beams. The large angle spreading is associated with the higher intensity probe beam used to study backward and forward scattering. The forward scattered light spectrum in dicate that classical or strongly driven Brillouin forward scattering is the primary mechanism for generating the density fluctuations related to the presence of the high intensity probe laser. Controlled fluctuations are studied in foam and exploding foil targets. The aim of these experiments is to investigate methods for imposing specific fluctuations on a laser-plasma. Controlled fluctuations using initial density inhomogeneities in the target are studied in foam targets with two different cell sizes. Forward scattered light measurements show that the initial foam target structure persists in the resulting plasma. Controlled fuctuations using a periodic intensity pattern are studied using exploding foil targets. Forward scattered light shows that the resulting density perturbation is correlated with the intensity pattern.

Modeling of the experiments is done with a wave kinetic equation which describes the propagation of laser light through a turbulent plasma. The kinetic equation is used to infer a fluctuation spectrum which describes the results in each experiment. The large scalelength and exploding foil plasma cases show that the 
fluctuation spectrum has characteristic length scales which correspond to the scalelengths of the transverse intensity variations in the high intensity lasers incident on the plasma. The foam case shows a fluctuation spectrum with length scales similar to the foam cell size. The main conclusions of the analysis are to characterize the intrinsic fluctuations in a large scalelength plasma and to show two methods for controling the scalesize of the fluctuations.

The remainder of this paper is divided into four sections. We describe the targets, laser systems, and diagnostics used in the different experiments in the next section, Section II. Section III describes the experimental results. Section IV describes the wave kinetic model and is applied to analyze the experimental results. Finally, Section V presents the conclusions.

\section{Experimental setup, targets, and instruments}

\section{A. Nova experiments}

The long scalelength plasma and foam experiments were conducted at the Lawrence Livermore National Laboratory using the Nova laser. This is a Nd:glass laser which operates at $1053 \mathrm{~nm}$ wavelength. The 10 Nova beams are positioned around a spherical target chamber. The beams are divided into two sets of five which impinge on each sidc of the target in an even distribution around a $50^{\circ}$ cone. The target is illuminated by 351-nm laser light that has been frequency converted from 1053-nm light. Unconverted 1053 and 527-nm light is blocked from striking the target but is present as stray light in the chamber. Each beam can produce up to about $3 \mathrm{~kJ}$ of $351-\mathrm{nm}$ light in a $1 \mathrm{~ns}$ constant power pulse.

The long scalelength plasma experiments used gasbag targets[ 8,9$]$. This target consists of two circular polyimide membranes attached to the edges of an aluminum washer. The region between the membranes is filled with a hydrocarbon gas to about 1 atm causing the membranes to expand outward in the shape of a balloon. The fill gasses consist of various $\mathrm{CH}$ mixtures. The resulting plasma density 
is varied between about $7.5 \%$ and $15 \%$ of critical density for 351 -nm light by changing the gas type and fill pressure. Argon dopant of $1 \%$ is added for spectroscopic measurements of temperature.

Gasbag plasmas have been extensively characterized with both experimental measurement and hydrodynamic simulation[ 10-12 ]. Gated x-ray pinhole camera measurements show that the gasbag becomes heated uniformly after about 0.3 to $0.4 \mathrm{~ns}$. The heater beams burn to the target center in about $0.3 \mathrm{~ns}$ and create a fairly uniform temperature plasma by 0.4 ns. The rapid blowdown of the gasbag polyimide 'skin' when the heater beams initially turn on launches a weak shock which propagates toward the center of the target leaving a rarefaction wave behind. Thomson scattering measurements show that the macroscopic plasma flow is small (about $4 \times 10^{6} \mathrm{~cm} / \mathrm{s}$ ) in the central region of the target but steadily increases outside the rarefaction wave. Helium- $\alpha$, Lyman- $\alpha$, and isoelectronic ratios obtained from $\mathrm{x}$-ray measurements are used to determine the lemporal evolulion of the electron temperature[ 12 ]. These mcasurements show that the central temperature gradually rises during the time that the heater beams are on to a peak of 2.5 to 3.0 $\mathrm{keV}$. Once the heaters turn off the temperature decreases as the plasma radiates and expands.

Nine of the Nova beams are used to heat the gasbag target and the tenth beam is used as a probe to study forward and backward scattered light. The gasbag laser setup is shown in Fig. 1 (a). The majority of the gasbag experiments described here used an $f / 8.5$ focus lens on the probe beam in order to simulate the laser characteristics from a 4-beamlet quad of NIF. This beam always passes through a kinoform phase plate (KPP) [ 13 ] located just after the final focus lens. The KPP spatially smoothes the intensity distribution in the focal spot producing a series of speckles in the laser far-field; the speckles have a width of $3 \mu \mathrm{m}(\sim f \lambda)$ and a length of about $200 \mu \mathrm{m}\left(8 f^{2} \lambda\right)$. The KPP also produces a nearly flat-top envelope to the laser intensity distribution at focus with an eliptical spot size of 260 by $400 \mu \mathrm{m}$. Two other laser smoothing techniques were implemented by conditioning the laser 
light prior to passing through the KPP. These techniques are called smoothing by spectral dispersion (SSD) [ 14-15] and polarization smoothing (PS)[16-19]. SSD was done by applying a $17 \mathrm{GHz}$ oscillating RF field to an electro-optic crystal to modulate the phase of the seed laser pulse. The frequency-broadened pulse was dispersed with a diffraction grating, amplified by the Nova laser amplifiers, and then focused onto the target. The resulting focal spot consists of many speckle patterns which interfere in time and space. A decorrelation time of about 5 ps is required for the speckle pattern to change substantially. Polarization smoothing was done by inserting a 2 by 2 array of birefringent glass between the conversion crystals and the final focus lens. This array was aligned so that the incident laser electric field vector was $45^{\circ}$ to both the ordinary and extraordinary axes of the birefringent glass. The glass is slightly wedged so that half of the energy in the incident beam produced a speckle pattern which was spatially shifted and orthogonally polarized to the speckle pattern formed from the other half of the energy. The smoothing effect from PS is instantaneous whereas the smoothing from SSD requires a decorrelation time for the speckle pattern to change significantly. The pulse-shape of the probe and heater beams is typically trapezoidal with about a 150 ps linear rise and fall and approximately constant power in between (typical variation is $15 \%$ ). Addition of SSD increases the rise time of the pulse to about 250 ps.

The foam targets were composed of either glass $\left(\mathrm{SiO}_{2}\right)$ or plastic $(\mathrm{CH})$. The glass foam, called aerogel, had an $0.5 \mu \mathrm{m}$ cell size (comparable to the $0.351 \mu \mathrm{m}$ laser wavelength) and the $\mathrm{CH}$ foam, called agar, had a much larger cell size of about $5 \mu \mathrm{m}$. Both foams had an average mass density of $3 \mathrm{mg} / \mathrm{cm}^{3}$. When fully ionized the agar foam gave an electron density of $11 \%$ critical for $351-\mathrm{nm}$ light and the aerogel foam was $9 \%$ critical electron density. Only the probe beam was used to heat and burn-through the foam for these experiments. The foam experimental setup is shown in Fig. 1 (b).

The primary forward scattered light instrument for the Nova experiments consisted of a high dynamic range two-part detector[20,21 ]. A scatter plate was used 
to measure the majority of the transmitted light. The plate had a central section measuring $45 \mathrm{~cm}$ in diameter which was surrounded by an annulus extending the overall angular coverage of the scatter plate to about $15^{\circ}$ from direct forward. The plate was composed of $\mathrm{SiO}_{2}$ that was roughened so as to give a uniform scattering surface.

The majority of the transmitted light that strikes the main scaller plate is measured using a fast photodiode and a gated optical imager (GOI). The photodiode is bandpass filtered from 345 to $355-\mathrm{nm}$ and has a rise time of $60 \mathrm{ps}$. Light scattered from the plate arrives at the photodiode over a spread of times due to the geometry of the plate and the viewing angle. The photodiode output is recorded by a scope giving an overall time response of about 250 ps. Deconvolving the plate response function from the diode signal using the light distribution on the plate improves the time response to about $150 \mathrm{ps}$.

The GOI takes three images of the 2-D light distribution on the scatter plate with a dynamic range of about 50:1. The gating time for an image is $200 \mathrm{ps}$. The images are taken at 300,600, and $900 \mathrm{ps}$ after the probe beam turns on. The images have a resolution at the scatter plate of about $1 \mathrm{~cm}$. Bandpass filters are also used on the three images of the GOI to exclude light outside the range $350-\mathrm{nm} \pm 5-\mathrm{nm}$.

The second part of the forward scattered light instrument is an array of halfinch diameter mirror detectors. These detectors measure the low level of light scattered at larger forward angles and are designed to have much higher dynamic range than the roughened scatter plate. The detectors consist of concave mirrors placed at increasing angles from direct forward. Nine of the detectors are mounted to one extension arm and 5 addional detectors are mounted to a second arm at a different azimuthal angle. Polarization of the incident laser is expected to give azimuthally dependent forward scattering which becomes more significant with increasing forward angle. The two sets of mirror detectors did not show a significant azimuthal difference indicating that the effect of polarization was not significant in these experiments. Light incident on the detectors is reflected by the mirror surface 
and directed across the target chamber to a port on the opposite side. The light passes through focus partway across the chamber and emerges through the chamber port at nearly a half-inch size spot. Light from all of the detectors is then imaged with a focusing optic onto a Spectralon diffuser plate and the time-integrated signal is recorded with two CCD cameras. A bandpass filter was used to transmit light within the spectral range of $350-\mathrm{nm} \pm 5-\mathrm{nm}$ into the cameras. Use of two cameras with a factor of 8 different intensity filtering increased the dyanmic range of the data. Mirror detectors at the smallest angles were uncoated and mirrors at larger angles were aluminized in order to give comparable signal levels.

In addition to the mirror detectors there are 6 fibers placed at a range of angles relative to the scatter plate. Two of the fibers are located behind the main scatter plate and 4 extend in angle out from it. The fibers carry light to the input of a 1-m spectrometer which disperses the light and images it onto the photocathode of a streak camera which records the time history of the spectrum. The output signal is spectrally resolved to about $0.7 \AA$ and temporally resolved to about $30 \mathrm{ps}$.

Measurements of the light transmitted from a 263-nm low intensity probe laser are made with $400 \mu \mathrm{m}$ optical fibers positioned at several places in the forward direction. We cannot spectrally resolve the small frequency shifts in the forward scattering of this light. Six fibers are placed from about $2^{\circ}$ to $10^{\circ}$. The light from the fibers passes through a 263-nm narrow bandpass filter and then onto the active surface of a photodiode. The fibers have staggered lengths so that the signals can be multiplexed onto one scope channel. The fiber transmission from detector to output is calibrated in-situ with a narrow-band filtered Xe source lamp. The diode is absolutely calibrated with a separate $263-\mathrm{nm}$ laser. The resulting signals shows the brightness of the forward scattered light from the 263-nm laser. The total incident energy of this beam is about $40 \mathrm{~J}$ in $1 \mathrm{~ns}$. The resulting intensity is about $1 \times 10^{14} \mathrm{~W} / \mathrm{cm}^{2}$ which is too low to perturb the local plasma parameters.

\section{Trident laser and target}


The exploding foil experiments were conducted using the Trident laser at the Los Alamos National Laboratory. The Trident laser is a Nd:glass laser that operates at 1053-nm. The laser has two beams which can achieve about $100 \mathrm{~J}$ of 1053-nm energy in 1-ns and a third beam which is used for probing purposes. This beam is typically operated at energies below $10 \mathrm{~J}$. The beams and target chamber are highly flexible allowing the facility to accommodate a wide range of beam and target geometries for various experiments. The experiments discussed here used the high power beams at the second harmonic (527-nm) in a 2-nm square pulse shape with $\mathrm{f} / 6$ focusing optics. The low power beam was operated in the 10 to $100 \mathrm{~mJ}$ range of third harmonic (351-nm) laser light in a $100 \mathrm{ps}$ pulse and was configured as an $\mathrm{f} / 100$ beam.

Exploding foil targets composed of 1 to $2.5 \mu \mathrm{m}$ of Mylar (CHO) were used to create the plasma. The experiments explored two heater beam configurations shown in Fig. 2 (a) and (b). In one case a wedge was placed in half of a single $\mathrm{f} / 6$ heater beam having $180 \mathrm{~J}$ in 2-ns of 527-nm light causing the beam to interfere with itself. The target was illuminated with a $500 \mu \mathrm{m}$ by $250 \mu \mathrm{m}$ focal spot having a $6.3 \mu \mathrm{m}$ intensity modulation. This beam burned through the foil and heated the resulting plasma to about $700 \mathrm{cV}$. At 1-ns a low cnergy $(10-100 \mathrm{~mJ}) \mathrm{f} / 100$ probe beam at 351-nm and a 100-ps wide Gaussian pulse was directed across the original surface of the foil in a transverse direction to the heater beam. The second heater beam configuration [Fig. 2 (b)] consisted of creating a higher spatial frequency modulation $(1.3 \mu \mathrm{m})$ in the intensity by interfering 2 separate heater beams, $23^{\circ}$ apart, at the target surface. The energy in each heater beam in this case was about $80 \mathrm{~J}$. The main diagnostic for this experiment consisted of an $\mathrm{f} / 1$ mirror placed near the target which collected the 351-nm scattered light. Additional optics imaged the surface of this mirror onto a CCD camera which recorded the data. Thomson scattering measurements diagnosed the density and temperature of this plasma.

\section{Experimental results}




\section{A. Nova gasbag experiments}

Figure 3 (a) and (b) show the time-integrated forward light signal for the 351$\mathrm{nm}$ beam with KPP smoothing only and then for KPP with SSD and PS for two plasma densities. The error bars indicate the uncertainty in the half-inch mirror detector calibration. The time resolved signal shows that most of the scattering occurs while both the heater and probe beams are on (from 0.5 to $1 \mathrm{~ns}$ ); the scattering amplitude decreases by about a factor of two to three after the heating pulse turns off. The ordinate in the plots is $\mathrm{J}_{\text {scat }} / \mathrm{sr}$ normalized to the incident beam energy. A no-target measurement would give a value of 90 within the $\mathrm{f} / 8.5$ cone (the value is typically 30 or less with a target). The data shows both small-angle and large-angle spreading. Small-angle spreading causes the incident f/ 8.5 beam to broaden by about a factor of 2 in angle (to $7^{\circ}$ ). 'T'he angular fall-off out to $7^{\circ}$ is determined using the measured 2-D gated image of the scatter plate. The energy fraction outside of the $7^{\circ}$ cone is about 15 to $30 \%$ of the total transmitted light for KPP smoothing only. Addition of SSD and PS beam smoothing reduces the large-angle scattered light and we infer that the associated short scalelength density fluctuations are also reduced. NIF baseline ignition simulations using beam spray ranging from what is measured here to about twice this reduces the fusion yield significantly. Adjusting the relative laser power in the inner and outer beam cones recovers high fusion yield. This shows that the ability to predict and tune-out the effects of beam spray is important for achieving ignition with the NIF.

Figure 4 shows the angular fall-off in the transmitted $263-\mathrm{nm}$ beam for two different plasma densities. The target density was varied by changing the gasbag pressure and gas mixture. The 263 -nm beam was pointed to pass through plasma not traversed by the 351-nm beam. We do not measure the angular dependence of the probe beam for $\theta<2.4^{\circ}$ so we show a constant value in this region based on an estimate (error of $\pm 10 \%$ ) of the probe transmission. We obtain this estimate by using the expression for inverse bremsstrahlung absorption and scaling the measured transmission of the $351-\mathrm{nm}$ beam. 
The spectrum of the scattered 351-nm beam has an angle-dependent red shift with a small amount of plasma-induced spectral broadening. The red shift at low intensity, shown in Fig. 5 (a) for f/4.3 and f/ 8.5 beams, increases with forward angle in a similar way as the classical SBFS[23-26] shift. This red-shift is given as $\left|\omega_{0}-\omega_{\mathrm{sc}}\right| /\left(k_{0} C_{\mathrm{s}}\right)=2 \sin (\theta / 2)$ where $\theta$ is the angle from the forward direction, $\omega_{0}$ is the probe frequency, $\omega_{\mathrm{sc}}$ is the scallered frequency, $k_{0}$ is the incident wavenumber, and $C_{s}$ is the ion sound speed determined from the measured backscattered red shift $\left(\Delta \lambda / \lambda=2.56 \times 10^{-3}\right.$ for $\left.\theta=180^{\circ}\right)$. Figure 5 (a) also shows the red shift for a high intensity $\mathrm{f} / 4.3$ beam at $\langle I\rangle \sim 8 \times 10^{15} \mathrm{~W} / \mathrm{cm}^{2}$. Strongly driven SBFS is the most likely explanation for the increased red shift since simple considerations can rule out explanations involving probe-plasma heating and self-phase modulation. The intensity required to give the increased red shift from strongly driven SBFS is estimated from the dispersion relation[ 22,23$]$ to be $I \sim 1 \times 10^{16} \mathrm{~W} / \mathrm{cm}^{2}$ for scattering at $20^{\circ}$. The high intensity $351-\mathrm{nm}$ beam has a large fraction of its energy in speckles at or above $1 \times 10^{16} \mathrm{~W} / \mathrm{cm}^{2}$. In light of this we conclude that classical or strongly driven SBFS is the primary physics process which creates the majority of the 351-nm forward scattered light beyond $7^{\circ}$. These measurements of spectra combined with the measured angular spreading can guide theoretical models of beam spray as well as help identify effective techniques for reducing beam spray.

Figure 5 (b) shows how the plasma-induced spectral width depends on angle and intensity. The spectrum is fit with a Gaussian and the width is defined as the Gaussian full width at half maximum (FWHM). The measured instrument resolution of $\Delta \omega / k_{0} C_{\mathrm{s}}=0.14(0.7 \AA)$ has been deconvolved from the values plotted in Fig. 5 (b). Plasma-induced bandwidth can result from finite beam width, filamentation, multiple scattering from SBFS, scattering from background density fluctuations, and damping of SBFS. There is no reason to rule out any of these at the present time and we must wait for further measurements to determine the relative importance of each effect. In spite of our uncertainty as to the origin of the bandwidth, we conclude that the bandwidth of the laser increases after traversing the 
plasma. This may be beneficial to suppressing growth of laser-plasma instabilities in the region beyond where the bandwidth develops[22 ].

\section{B. Foam experiments}

Figure 6 shows electron micrographs of the two foams studied in the forward scattering experiment. The length scale between fibers of the foam has some variability but on average the agar $(\mathrm{CH})$ has a large spacing of about $5 \mu \mathrm{m}$ and the aerogel has a small spacing of $0.5 \mu \mathrm{m}$. The aerogel spacing is close to the 0.351 $\mu \mathrm{m}$ laser light wavelength and is therefore expected to scatter the light by a larger angle than the agar. Only one Nova beam is used for the experiment so that the forward scattered light signal is weaker due to substantial energy absorption during the initial heating process. The angular distribution of light scattered from the agar foam ( $5 \mu \mathrm{m}$ cell size) falls off rapidly with increasing forward angle as shown in Fig. 7. On the other hand, the aerogel foam (0.5 $\mu \mathrm{m}$ cell size) shows a nearly constant level of angular spread light as far out as the last detector at $35^{\circ}$.

The spectral data for the foams shows a broad essentially unshifted signal as opposed to a red or blue shifted signal. This spectral information indicates that the scattering is from the foam structure rather than being excited by forward scattering instabilities.

\section{Trident foil experiments}

The first heater beam configuration has a wedge in half of one heater beam causing the two half-beams to interfere at the target. We checked the resulting intcrference pattcrn by dirccting an alignment beam through the beam geometry and inspecting the resulting interference pattern with a CCD camera. The separation between intensity maxima is $6.3 \mu \mathrm{m}$ in this configuration and the two half-beams overlap to give a spot measuring about $500 \mu \mathrm{m}$ by $250 \mu \mathrm{m}$. The $0.351 \mu \mathrm{m}$ probe beam, which propagates transversely to the heater beam, is expected to be deflected in two directions by $3.2^{\circ}$ based on $k$-matching the probe and the expected imposed density fluctuation. 
Figure 8 shows the forward scattered light from the probe beam when the foil is illuminated with a self-interfering heater beam (a) and when the wedge is removed giving no interference pattern (b). Removing the wedge gives a circular spot on the target measuring $500 \mu \mathrm{m}$ in diameter. To keep the average intensity of the circular spot (no wedge) similar to the spot with the wedge, the beam focus was adjusted decreasing the circular focal spot to a $350 \mu \mathrm{m}$ diameter. The bright straight-through light in both images, shown in Fig. 8, is blocked with a thin obscuration placed on the collection mirror. Figure 8 (a) shows signal above and below the location of the target at equal angles corresponding to scattering at $3.1^{\circ}$. Removal of the wedge causes this portion of the light to vanish as seen in Fig. 8 (b). Installing and removing the wedge was repeated a number of times and each time we found the same result. The additional bright light emerging from the obscuration is light refracted by the exploding foil plasma. This is not filamentation-incduced beam bending because the beam intensity is too low to filament and/or backscatter ( $I \leq$ $\left.1 \times 10^{13} \mathrm{~W} / \mathrm{cm}^{2}\right)$. The energy of light scattered into the spots at $\pm 3.1^{\circ}$ is estimated to be $1.2 \times 10^{-4}$ of the main incident energy.

The second heater beam configuration did not show evidence of probe laser scattering; the scattering angle in this case is $15.3^{\circ}$. This configuration should produce an interference pattern on the target with a separation of $1.3 \mu \mathrm{m}$ between intensity maxima. We were not able to verify the interference pattern at the target but verified that the beams overlapped, that they arrived at the target simultaneously, that they were temporally coherent at the target, and that their polarizations were aligned. We did not observe any scattered light fraction above $10^{-5}$.

\section{Modeling}

The goal in modeling these experiments is to infer from the forward scattered light a plasma density fluctuation (PDF) spectrum which, when used in our scattering model, gives a calculated forward scattered light signal which matches the measured forward scattered light signal. We do not attempt to describe the detailed 
process whereby filamentation/SBFS or other low frequency processes lead to this fluctuation spectrum.

The fluctuations causing the observed forward scattering are low-frequency $\left[\left|\omega-\omega_{0}\right| / \omega_{0}<9 \times 10^{-4}\right.$ from Fig. 5 (a) $]$ so they can be considered stationary for the purpose of calculating the angular scattering. The method for inferring the fluctuation spectrum is to first prescribe the shape and amplitude of a stationary PDF spectrum $S(k)$. The spectral amplitude is normalized to a mean-square fluctuation level, defined as $\left\langle|\delta n / n|^{2}>\equiv \int S(k) d^{3} k\right.$, where the plasma density is written as $n_{e}=n+\delta n$ and $n$ is the avcrage clcctron density. Next, we compute the scattered light distribution resulting from this PDF spectrum. This is done using a wave kinetic equation which we describe below.

An electromagnetic wave propagating through a plasma with a varying density evolves according to the wave equation

$$
\nabla^{2} E-\frac{1}{c^{2}} \frac{\partial^{2} E}{\partial t^{2}}=\frac{\omega_{p 0}^{2}}{c^{2}}\left(1+\delta n / n_{0}\right) E
$$

where $c$ is the speed of light and the nonuniform density, $\delta n$, only varies in space and not in time. The only time dependence enters through the frequency of the electric field which is written as $E(r, t)=E(r) \exp \left[-i \omega_{0} t\right]$ where $\omega_{0}$ is the oscillation frequency of the field. Since we are assuming stationary fluctuations $\omega_{0}$ is the only frequency in the problem. We assume that the scattering is weak so that power is scattered out of the main beam slowly compared to the rapid variation in the phase of the electric field along the direction of propagation which we take to be $z$. This allows us to make the envelope approximation and write the electric field as $E(r)=E(x, y, z) \exp \left[i k_{0} z\right]$. Making the paraxial approximation and assuming that diffraction can be neglected gives the first order equation for the envelope, $E$, which is written as

$$
\frac{\partial E}{\partial z}=\frac{-i}{2 k_{0}} \frac{\omega_{p}^{2}}{c^{2}} \frac{\delta n}{n_{0}} E .
$$


This equation has the formal solution

$$
E(z)=E_{0} \exp \left[\frac{-i}{2 k_{0}} \frac{\omega_{p}^{2}}{c^{2}} \int_{0}^{z} d z^{\prime} \frac{\delta n}{n_{0}}\right] .
$$

This solution for the electric field shows that the plasma creates a perturbation to the electric field phase which transforms to an intensity perturbation after the light propagates far away from the plasma to the location of the detectors. This process is shown schematically in Fig. 9.

Equation (4) is not very useful since it describes the electric field and the measurement is made of power or energy. Furthermore, there are many locations in the plasma which scattcr light to the detectors. Each of these scattering locations is statistically independent from the other making the scattered light equal to an ensemble sum of random phased scatterers. This kind of scattering is calculated with a wave kinetic equation that describes how the power is scattered by an ensemble of random phased scatterers. Following a similar procedure as outlined in References ?? we use Eq. (3) to derive a wave kinetic equation for the ensemble sum of the power spectrum $P_{i j}(z)$ at position $z$ through the plasma. This power is expressed in terms of the electric field as

$$
P_{i j}(z)=\frac{c}{8 \pi}\left|E_{i j}(z)\right|^{2}
$$

where

$$
E_{m, n}(z)=\frac{1}{L_{x}} \frac{1}{L_{y}} \int d x \int d y E(x, y, z) \exp \left(-i k_{x m} x-i k_{y n} y\right) .
$$

and $k_{x m}=2 \pi m / L_{x}, k_{y n}=2 \pi n / L_{y}$, and $m$ and $n$ are integers. The kinetic equation is written as

$$
\frac{d P_{i j}}{d z}=\left(\frac{1}{2 k_{0}} \frac{\omega_{p}^{2}}{c^{2}}\right)^{2}\left[\sum_{l m} P_{i-l, j-m} S_{l m}-\sum_{u v} P_{i j} S_{u-i, v-j}\right]
$$

where $S_{i j}$ is the 2-D surface within the spectral energy density of the fluctuations for which the magnitude of the light wave $k$ is constant. If the laser $|k|$ is large compared 
to the $k$-values for which $S(k)$ is significant then this surface is essentially a plane given by $S_{i, j, l=0}$. The density fluctuation amplitude is related to $S_{i, j, l}$ as

$$
<|\delta n / n|^{2}>=\sum_{i j l} S_{i j l}
$$

The first term in Eq. (6) for $d P_{i j} / d z$ represents the rate at which power is scattered from all of the other spectral components into $P_{i j}$ and the second term describes the rate at which power is scattered out of $P_{i j}$. It is possible to solve this kinetic equation in closed form as

$$
\begin{aligned}
P_{i j}(z) & =\sum_{l m} P_{0 i-l, j-m} \frac{1}{L_{x} L_{y}} \int d x d y \exp \left(-i k_{x l} x-i k_{y m} y\right) \\
& \exp \left[z \frac{k_{0}^{2}}{4}\left(\frac{n}{n_{c}}\right)^{2}\left(\bar{S}(x, y)-S_{0}\right)\right]
\end{aligned}
$$

where

$$
\bar{S}(x, y)=\sum_{p q} S_{p q} \exp \left(-i k_{x p} x-i k_{y q} y\right)
$$

$S_{0}=\bar{S}(x=0, y=0), P_{0 i j}$ is the incident power, and $z$ is the propagation distance in the plasma.

The equation for $P_{i j}(z)$ is used to model the experiment as follows. A fluctuation spectrum is guessed which has characteristics suggested by the forward scattered light. This spectrum is then used to calculate a power distribution of laser light after propagating through the plasma. The calculated angular spread is compared with the data and the fluctuation spectrum is adjusted to bring the best agreement between the calculated and measured angular powers. This technique provides a way to determine the general characteristics of the fluctuation spectrum. The actual numerical calculation of the angular spread for the laser light is done using either a Monte Carlo ray tracing calculation of the wave kinetic equation [Eq. (6)] or by computing $P_{i j}(z)$ from Eq. 8. The Monte Carlo calculation follows the ray trajectories and is not constrained to be paraxial. A disadvantage of the Monte Carlo technique is that many rays are required to achieve good statistics at 
larger angles where the amount of scattered light is small. There is not a significant difference between calculations of the forward scattered light using either technique for this experiment.

\section{B. Gasgab results}

The gasbag results show evidence of both small and large-angle scattering which can only be explained using a two component fluctuation spectrum. We constructed a spectrum having two components and then varied the parameters describing the spectrum so as to match the data. The spectrum has a constant value between $0 \leq k \leq k_{s}$ (small- $k$ part) then it changes to a different constant value for $k_{s} \leq k \leq k_{l}$ (large- $k$ part). This spectum is defined analytically using 4 parameters: 1) the mangitude of $\langle|\delta n / n|\rangle, 2$ ) the extent of the small- $k$ part of the spectrum $\left.\left(k_{s}\right), 3\right)$ the extent of the large- $k$ part of the spectrum $\left(k_{l}\right)$ and 4$)$ the ratio of the large- $k$ and small- $k$ part. A parameter scan of these quantities led to the spectrum shown in Fig. 10 (a) which best fits the data at low density. It is difficult to fit the high density data with this simple two-part constant spectrum. We found that we could match the data if we used an decreasing exponential rather than a constant value for the large- $k$ part, this is shown in Fig. 10 (b). It is also possible to fit the data at low density using a decreasing exponential. Comparing the two types of spectra we see that both exhibit the basic features of a small- $k$ part and a large- $k$ part; the main distinction is the transition between the two spectral regions. We have taken the spectrum in the calculation to be isotropic for the following reasons. The background spectrum appears to come from the heater beams; since these beams are approximately isotropic we expect the density perturbations created by them to also be isotropic. The probe-induced density perturbations seem to arise chiefely from SBFS. This instability grows exponentially in space so that the largest amplitude correllated density perturbation due to the ion wave exists mainly in the last e-fold of growth. This is a localized region which we've taken to be on the order of an $\mathrm{f} / 8$ speckle width. There is no data available to indicate the true longitudinal 
correlation length of the fluctuations. If the correlation length is longer than an $f / 8$ speckle width (about $4 \mu \mathrm{m}$ ) then the density fluctuation amplitude will be reduced. For a fixed scattered power the fluctuation amplitude scales as $1 / s q r t l_{c}$ where $l_{c}$ is the longitudinal correlation length.

The same wave-kinetic equation used to predict the angular spreading for the 351-nm beam is used to calculate the spreading of the 263-nm beam. We find that using only the small- $k$ part of the spectrum found in the 351-nm case can explain the measured angular fall-off of the 263-nm light. Comparison of the calculated scattered light and the measured light shows good agreement at both low and high electron density as seen in Fig. 4.

\section{Foam results}

Applying the same wave kinetic model to the foam results leads to the two spectra shown in Fig. 11 Both foams show a spectrum that roughly matches the scalesize of the initial density perturbations given by the foam cell size.

The duration of the signal is about $600 \mathrm{ps}$ for the aerogel foam and about $300 \mathrm{ps}$ for the agar foam. We explain this duration as follows. The large density structures in the foams smooth out with an ion acoustic decay time scale. The hydrogen gives the agar foam $(\mathrm{CH})$ a larger ion acoustic damping compared to the aerogel for a specific ion acoustic wavelength. Due to the large difference in cell sizes the damping time for the longer ion acoustic wave $(5 \mu \mathrm{m})$ in the agar is the same as the damping time for the $0.5 \mu \mathrm{m}$ wavelength wave in the aerogel, which is 33 ps. The density perturbations may actively scatter for about 100 ps before the scattered light level becomes insignificant. In addition, the single interaction beam takes 200 to 400 ps to burn through the foam depending on the precise thickness (about 1 to $1.5 \mathrm{~mm}$ ). The sum of the decay time and burn-through time total the duration over which signal is observed.

\section{Exploding foil results}


The first heater beam configuration used the wedge to construct an interference pattern at the target. The angle of interference between the two beam halves is the half-angle of an $\mathrm{f} / 6$ beam or $4.8^{\circ}$. The resulting electric field at the target for perfect beams is proportional to $\exp \left[i k_{0} x \cos (1 / 24)+i k_{0} y \sin (1 / 24)\right]+$ $\exp \left[i k_{0} x \cos (1 / 24)-i k_{0} y \sin (1 / 24)\right]$. The intensity is given by $4 I_{0} \sin ^{2}\left[k_{0} y \sin (1 / 24)\right]$ which has a modulation period in the $y$ direction (transverse to the beam propagation direction) of $6.3 \mu \mathrm{m}$ for $0.527 \mu \mathrm{m}$ laser light. The plasma is expected to respond to the modulated ponderomotive force. The actual beams have inherent intensity structure so that the correlation length along the $x$ direction of the interference pattern is about $100 \mu \mathrm{m}$. Additional plasma nonuniformities can further reduce the correlation length of the interference pattern. The probe beam has a $0.351 \mu \mathrm{m}$ wavelength and propagates transversely to the $6.3 \mu \mathrm{m}$ variation in the interference pattern. The scattering angle obtained by $k$-matching the probe with the interference pattern is equal to $3.1^{\circ}$. This is the expected angle for scattering light in both directions along the intensity variation.

Figure 8 (a) shows a forward scattered light signal at both $+3.1^{\circ}$ and $-3.1^{\circ}$ with the wedge installed. Removing the wedge causes the scattered light at the $\pm 3.1^{\circ}$ to vanish as seen in Fig. 8 (b). The measured angle agrees with the expected scattering angle within the measurement error. This result gives evidence that the intensity pattern scalelength due to the wedge is impressed on the plasma with the same scalelength.

Figure 8 (a) also shows that the scattered light amplitude (estimated from the measured signal and a calibration signal) normalized to the incident energy is $1.2 \times 10^{-4}$. This level of light is used in the scattering model to make an estimate of the amplitude of $\langle|\delta n / n|>$ for a given longitudinal correlation length of the density perturbation. The parameters for the calculation are as follows. The plasma density is taken to be $5 \%$ of critical for 527 -nm light ( $2.2 \%$ critical for $351-n m$ ), the $351-\mathrm{nm}$ probe is $\mathrm{f} / 100$ and is directed so as to pass over the surface of the foil. The 527 -nm heater beam is normal to the foil and creates an interference pattern with 
a wavelength of $6.3 \mu \mathrm{m}$ directed tangentially to the foil surface and transversely to the 351-nm probe beam. This pattern is estimated to remain correlated over $60 \mu \mathrm{m}$ in the plane normal to the probe beam and $90 \mu \mathrm{m}$ in the direction of the probe. Figure 12 (a) shows the calculation of the scattered light for $\langle|\delta n / n|\rangle=0.002$; this gives the same scaltered light amplitude as measured in the experiment. If the scattered light fraction is small compared to unity it is proportional to the product $\left(k_{0} L_{z}\right)\left(k_{0} l_{c}\right)\left(n / n_{c}\right)^{2}<|\delta n / n|^{2}>$ where $L_{z}$ is the length of the overall region where scattering occurs and $l_{c}$ is the longitudinal correlation length of the density perturbation. This expression can be used to scale the actual density perturbation for different values of $l_{c}$ or $L_{z}$. Figure 12 (b) shows the density fluctuation power spectrum used in the calculation of the scattered light in Fig. 12 (a).

The expected density perturbation due to the ponderomotive pressure from the imposed intensity pattern is given as $0.25 v_{o s}^{2} / v_{t h}^{2}$ where $v_{o s}$ is the quiver velocity of the electrons and $v_{t h}$ is the electron thermal velocity. This can bc written as $\delta n / n \sim 0.02\left[I / 10^{14} \mathrm{~W} / \mathrm{cm}^{2}\right]\left[0.5 / T_{c}(\mathrm{keV})\right][\lambda / 1.054 \mu \mathrm{m}]^{2}$. The intensity at the peaks in the pattern is approximately $1 \times 10^{14} \mathrm{~W} / \mathrm{cm}^{2}$. The steady-state density perturbation estimated for a ponderomotive pressure is given as $\delta n / n \sim$ $0.02\left[1 / 10^{14} \mathrm{~W} / \mathrm{cm}^{2}\right]\left[0.5 / T_{e}(\mathrm{keV})\right][\lambda / 1.054 \mu \mathrm{m}]^{2}$. Using $0.527 \mu \mathrm{m}$ for the wavelength and $0.7 \mathrm{keV}$ for the electron temperature (estimated from Thomson scattering) gives $\delta n / n=4 \times 10^{-3}$ which is within a factor of two of the estimate using the scattering model. This indicates that our estimates for the correlation lengths may be reasonable; a factor of two error in the density fluctuation amplitude corresponds to a factor of 1.4 in the correlation lengths or propagation distance.

The second heater beam configuration did not produce any measurable scattered light at the expected angle. This configuration used two $\mathrm{f} / 6$ heater beams at $23^{\circ}$ center-to-center which form an interference pattern with a $1.3 \mu \mathrm{m}$ peak-topeak spacing. A density perturbation with this scalesize impressed in the plasma is expected to give a scattering angle of $15.3^{\circ}$ for the $0.351 \mu \mathrm{m}$ probe beam. During the experiment we confirmed that the beam timing and polarization between the 
two heater beams were correct for creating an interference pattern. We varied the intensity of the heater spot by varying the energy in the beams and by changing the spot size. None of these changes led to a clear scattered light signal at $15.3^{\circ}$.

There are several reasons we have considered which may account for this result. The heater beams may loose enough coherence due to the phase perturbations introduced by the plasma that the interference pattern does not form with much spatial coherence. As a result, the scattered light amplitude may be significantly reduced. Another possibility is that $k$-matching may not be sufficiently satisfied in this geometry resulting in a reduced scattering efficiency. The density perturbation $k$ vector is oriented $90^{\circ}$ relative to the probe beam $k$ vector. Exact $k$ matching is achieved by having the angle between the probe beam and the density perturbation $k$ vectors of $97.7^{\circ}$ or $82.3^{\circ}$. The experimental setup made it difficult to test this in the time allotted for the experiment.

\section{Summary}

We have described experiments investigating intrinsic and controlled fluctuations in different types of laser-produced plasmas. The aim of the gasbag experiments was to study the fluctuation spectrum and forward scatter which results from 9 heater beams and one higher intensity probe beam illuminating a plasma having similar density and scalelength as the central region of a NIF hohlraum. The results show small angle and large angle beam spread in the forward direction. This can only be explained with a density fluctuation spectrum having small and large- $k$ components. Spectral measurements indicate that the forward scattering arises from stimulated Brillouin forward scattering (SBFS) and filamentation. A passive scattering model shows agreement with the measurements for a fluctuation

amplitude of $\langle|\delta n / n|>=0.08$. Adding SSD and polarization smoothing leads to a reduction in the large angle scattering giving $\langle|\delta n / n|\rangle=0.06$. This almost recovers the background level which is separately determined to be $\langle|\delta n / n|\rangle=0.05$ using the forward scatter from the 263-nm low intensity probe beam. 
Experiments using foam targets with two different cell sizes show evidence of target structure in the resulting plasma which can be observed with forward scattered light. Precise time-dependent measurements of forward scattered light may be useful for determining the rate at which the initial density perturbations smooth out in a foam target. Analysis of the data shows that the scattered light can be explained using fluctuation spectra with scalelengths similar to the initial cell size of the foams.

Exploding foil experiments using the Trident Laser show that an intensity pattern creates a correllated density perturbation with an estimated amplitude that agrees with simple ponderomotive force estimates. This result confirms our understanding of how a moderate intensity laser creates a density perturbation. This result can be useful for developing more sophisticated models of laser propagation through a plasma.

The author gratefully acknowledges useful discussions with B. B. Afeyan, S. N. Dixit, D. S. Montgomcry, S. M. Pollaine, A. M. Rubenchik and the help of R. Griffith with the streak camera instrument. This work is performed under the auspices of the U.S. Department of Energy by the Lawrence Livermore National Laboratory under Contract No. W-7405-ENG-48. 


\section{REFERENCES}

1. A. V. Maximov, W. Rozmus, et al., Phys. Plasmas 3, 1689 (1996).

2. B. B. Afeyan, A. E. Chou, et al., Phys. Rev. Lett. 80, 2322 (1998).

3. D. S. Montgomery, B. B. Afeyan, et al., Phys. Plasmas 5, 1973 (1998).

4. J. D. Lindl, Phys. Plasmas 2, 3933 (1995).

5. S. W. Haan, S. M. Pollaine, J. D. Lindl, L. J. Suter, R. L. Berger, L. V. Powers, W. E. Alley, P. A. Amendt, J. A. Futterman, W. K. Levedahl, M. D. Rosen, D. P. Rowley, R. A. Sacks, A. I. Shestakov, G. L. Strobel, M. Tabak, S. V. Weber, G. B. Zimmerman, W. J. Krauser, D. C. Wilson, S. V. Coggeshall, D. B. Harris, N. M. Hoffman, and B. H. Wilde, Phys. Plasmas 2, 2480 (1995).

6. R. L. Kauffman, L. J. Suter, C. B. Darrow, J. D. Kilkenney, H. N. Kornblum, D. S. Montgomery, D. W. Phillion, M. D. Rosen, A. R. Theissen, R. J. Wallace, and F. Ze, Phys. Rev. Lett. 73, 2320 (1994).

7. S. H. Glenzer, F. B. Rosmej, R. W. Lee, C. A. Back, K. G. Estabrook, B. J. MacGowan, T. D. Shepard, and R. E. Turner, Phys. Rev. Lett. 81, 365 (1998).

8. B. J. MacGowan, B. B. Afeyan, C. A. Back, R. L. Berger, G. Bonnaud, M. Casanova, B. I. Cohen, D. E. Desenne, D. F. DuBois, A. G. Dulieu, K. G. Estabrook, J. C. Fernandez, S. H. Glenzer, D. E. Hinkel, T. B. Kaiser, D. H. Kanantar, R. L. Kaufman, R. K. Kirkwood, W. L. Kruer, A. B. Langdon, B. F. Lasinski, D. S. Montgomery, J. D. Moody, D. H. Munro, L. V. Powers, H. A. Rose, C. Rousseaux, R. E. Turner, B. H. Wilde, S. C. Wilks, and E. A. Williams, Phys. Plasmas 3, 2029 (1996).

9. D. H. Kalantar, D. E. Klem, B. J. MacGowan, J. D. Moody, D. S. Montgomery, D. H. Munro, T. D. Shepard, and G. F. Stone, Phys. Plasmas 2, 3161 (1995).

10. J. J. Denavit and D. W. Phillion, Phys. Plasmas 1, 1971 (1994).

11. L. V. Powers, R. L. Berger, R. L. Kauffman, B. J. MacGowan, P. A. Amendt, C. A. Back, T. P. Bernat, S. N. Dixit, D. I. Eimerl, K. G. Estabrook, J. A. Harte, D. H. Kalantar, D. E. Klem, B. F. Lasinski, D. S. Montgomery, J. D. Moody, D. H. Munro, T. D. Shepard, L. J. Suter, R. E. Turner, and E. A. Williams, Phys. Plasmas 2, 2473 (1995).

12. S. H. Glenzer, C. A. Back, K. G. Estabrook, B. J. MacGowan, D. S. Montgomery, R. K. Kirkwood, J. D. Moody, D. H. Munro, and G. G. Stone, Phys. Rev. E 55, 927 (1997). 
13. S. N. Dixit, J. K. Lawson, K. R. Manes, H. T. Powell, and K. A. Nugent, Optics Letters 19, 417 (1993); S. N. Dixit, M. D. Feit, M. D. Perry, and H. T. Powell, Optics Letters 21, 1715 (1996).

14. S. N. Dixit, I. M. Thomas, B. W. Woods, A. J. Morgan, M. A. Henesian, P. J. Wegner, and H. T. Powell, Appl. Opt. 32, 2543 (1993); J. W. Goodman, Statistical Oplics (Wiley, New York, 1985).

15. S. Skupsky, R. W. Short, T. Kessler, R. S. Craxton, S. Letzring, and J. M. Soures, J. Appl. Phys. 66, 3456 (1989).

16. "Phase Conversion Using Distributed Polarization Rotation," LLE Review 45, 1-12 (1990).

17. K. Tsubakimoto, M. Nakatsuka, H. Nakano, T. Kanabe, T. Jitsuno, and S. Nakai, Opt. Commun. 91, 9-12 (1992); K. Tsubakimoto, T. Jitsuno, N. Miyanaga,

M. Nakatsuka, T. Kanabe, and S. Nakai, Opt. Commun. 103, 185-188 (1993).

18. S. Pau, S. N. Dixit, and D. Eimerl, J. Opt. Soc. Am. B 11, 1498 (1994).

19. E. Lefebvre, R. L. Berger, A. B. Langdon, B. J. MacGowan, J. E. Rothenberg, and E. A. Williams, Phys. Plasmas 5, 2701 (1998).

20. J. D. Moody, B. J. MacGowan, B. B. Afeyan, S. H. Glenzer, R. K. Kirkwood, W. L. Kruer, S. M. Pollaine, A. J. Schmitt, E. A. Williams, and G. F. Stone, Review of Scientific Inst. 68, 1725 (1997).

21. J. D. Moody, B. J. MacGowan, B. B. Afeyan, S. H. Glenzer, R. K. Kirkwood, W. L. Kruer, S. M. Pollaine, A. J. Schmitt, E. A. Williams, and G. F. Stone, Rev. Sci. Inst. 70, 677 (1999).

22. A. J. Schmitt and B. B. Afeyan, Phys. Plasmas 5, 503 (1998).

23. J. F. Drake, P. K. Kaw, et al., Phys. Fluids 17, 778 (1974).

24. C. J. McKinstrie, J. S. Li, and A. V. Kanaev, Phys. Plasmas 4, 4227 (1997); Lal, et al., Phys. Rev. Lett 78, 670 (1997).

25. V. V. Eliseev, et al., Phys. Plasmas 4, 4333 (1997).

26. C. Labaune, et al., Bull. Am. Phys. Society 43, 1639 (1998). Also, submitted to Physics of Plasmas November 1998. 


\section{FIGURE CAPTIONS}

FIG. 1 (a) Schematic of the detection system for measuring the amplitude and spectra of the near forward scattered light. The $\mathrm{f} / 8.5$ beam is converted to an $\mathrm{f} / 4.3$ beam in some cases. (b) Experimental setup for the foam measurements.

FIG. 2 Experimental setup for the exploding foil experiments using the Trident laser facility for (a) the small angle between the beams $\left(4.8^{\circ}\right)$ and (b) the larger angle $\left(23^{\circ}\right)$ between the beams.

FIG. 3 Measurement of the forward scattered light amplitude vs angle for a (a) $7.5 \%$ critical plasma and (b) a $14 \%$ critical plasma.

FIG. 4 Angular fall-off of the forward scattered light from the 263-nm lowintensity probe beam shown with the calculated fall-off for $\langle|\delta n / n|\rangle=0.05$.

FIG. 5 (a) Red shift and (b) spectral width of the measured forward scattered light from a $7 \%$ critical density gasbag plasma.

FIG. 6 Electron micrograph of (a) agar fuam and (b) aerogel foam.

FIG. 7 Plot of angular fall-off of scattered light from the two different cell size foams.

FIG. 8 Measurements of the Trident experiment showing (a) the scattered light a.t $3.1^{\circ}$ with the wedge in and (b) the absence of the scattered light when the wedge is removed.

FIG. 9 Schematic shows how the plasma alters the laser phase as it propagates through an inhomogeneous plasma density.

FIG. 10 Spectrum used to best-fit the measured forward scattered light for (a) a $7.5 \%$ critical density gasbag plasma and (b) a $15 \%$ critical plasma.

FIG. 11 Spectrum used to fit the forward scattered light data for (a) the agar foam and (b) the aerogel foam.

FIG. 12 (a) Calculation of the scattered light for the Trident experiment using $<|\delta n / n|>=0.002$ and the spectrum shown in (b). 


\section{Figure 1 (a)}

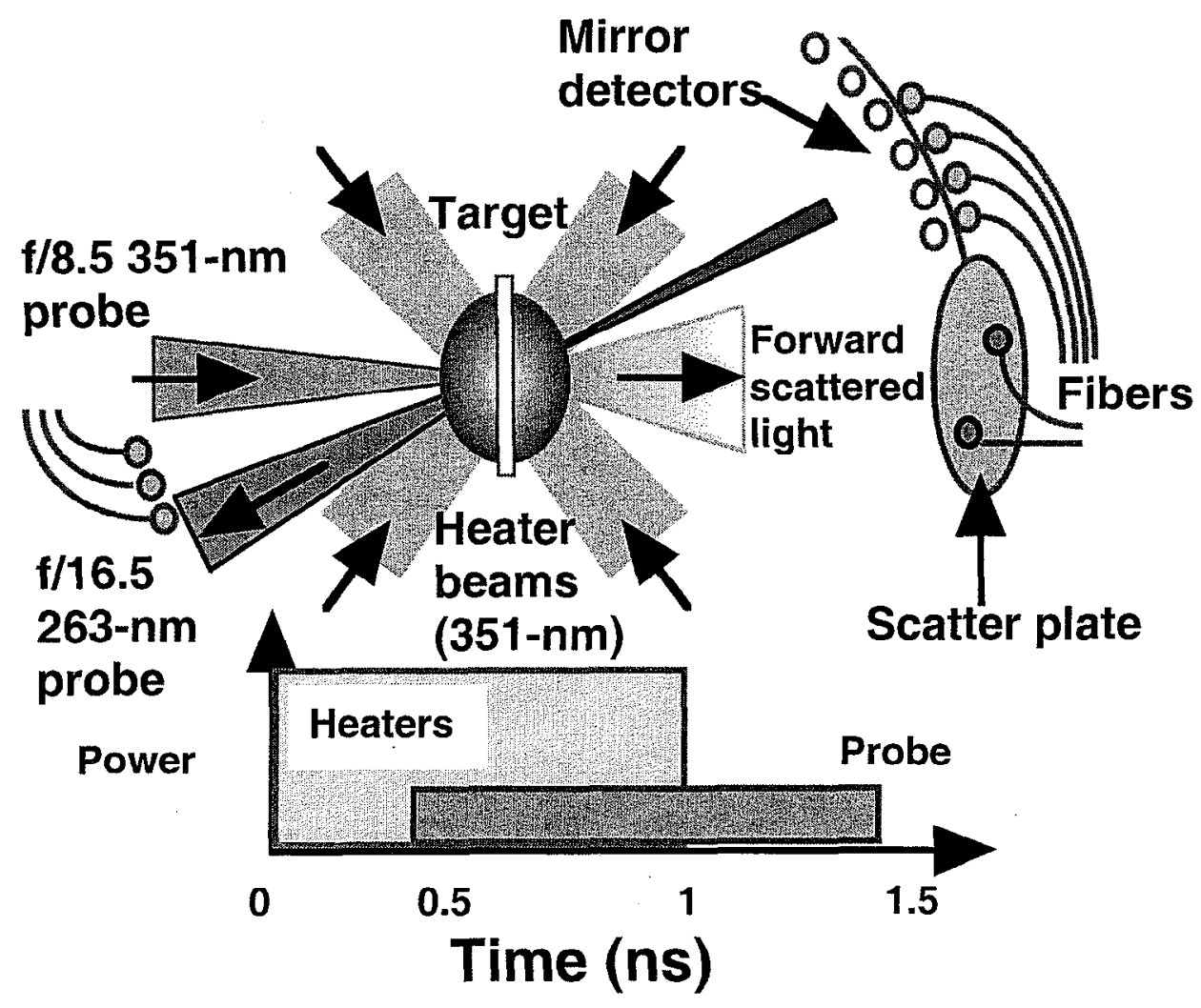


Figure 1 (b)

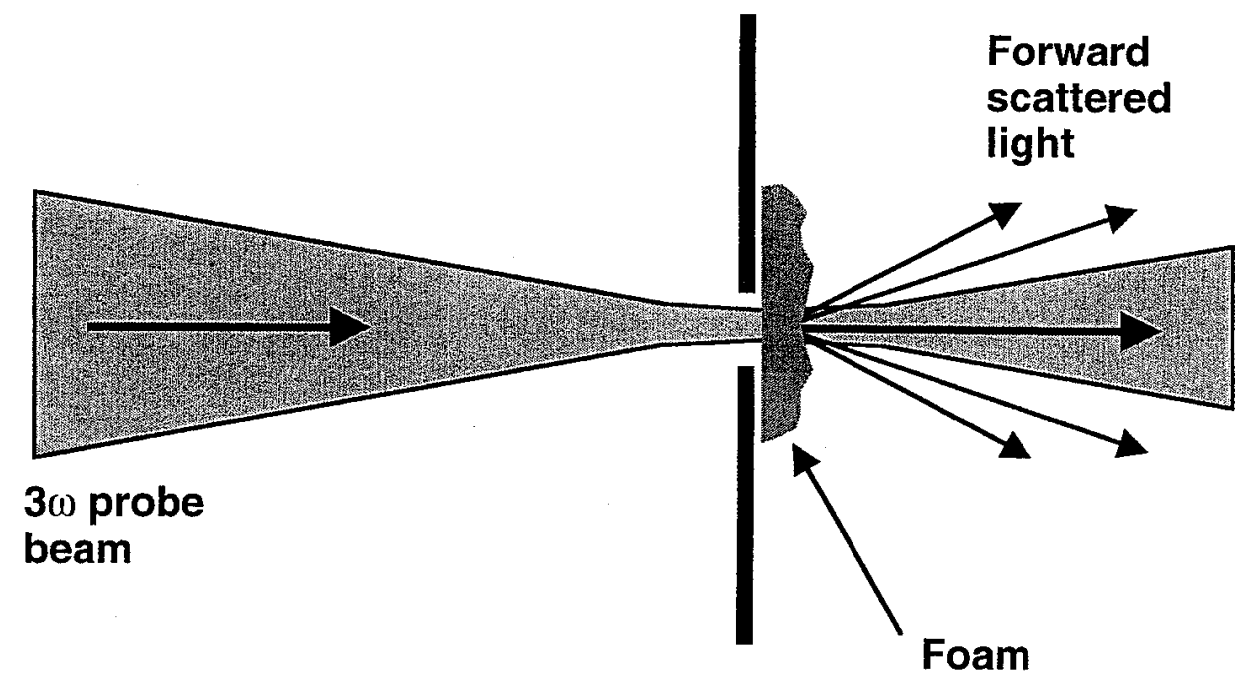


Figure 2 (a)

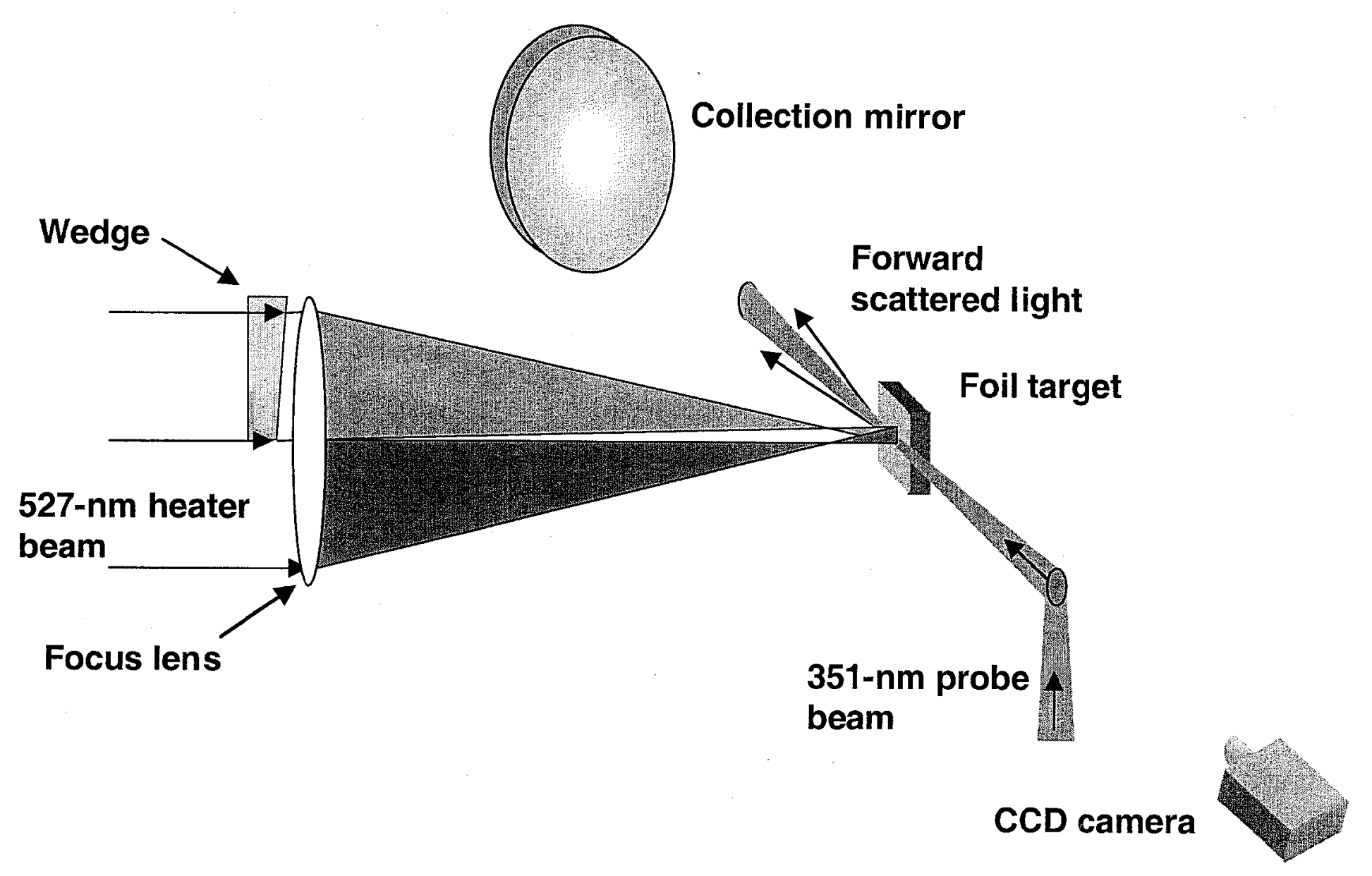




\section{Figure 2 (b)}

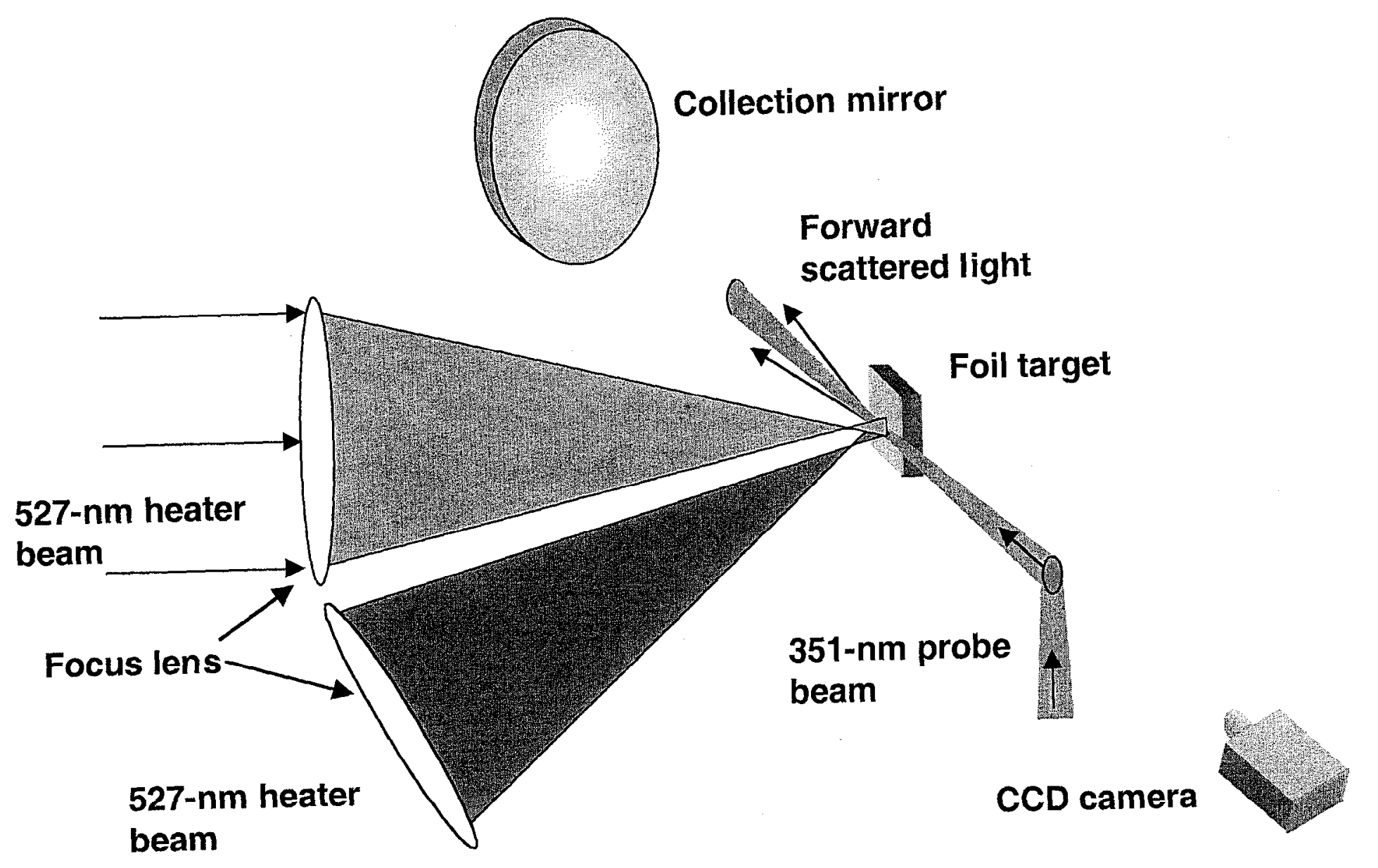


Figure 3 (b)

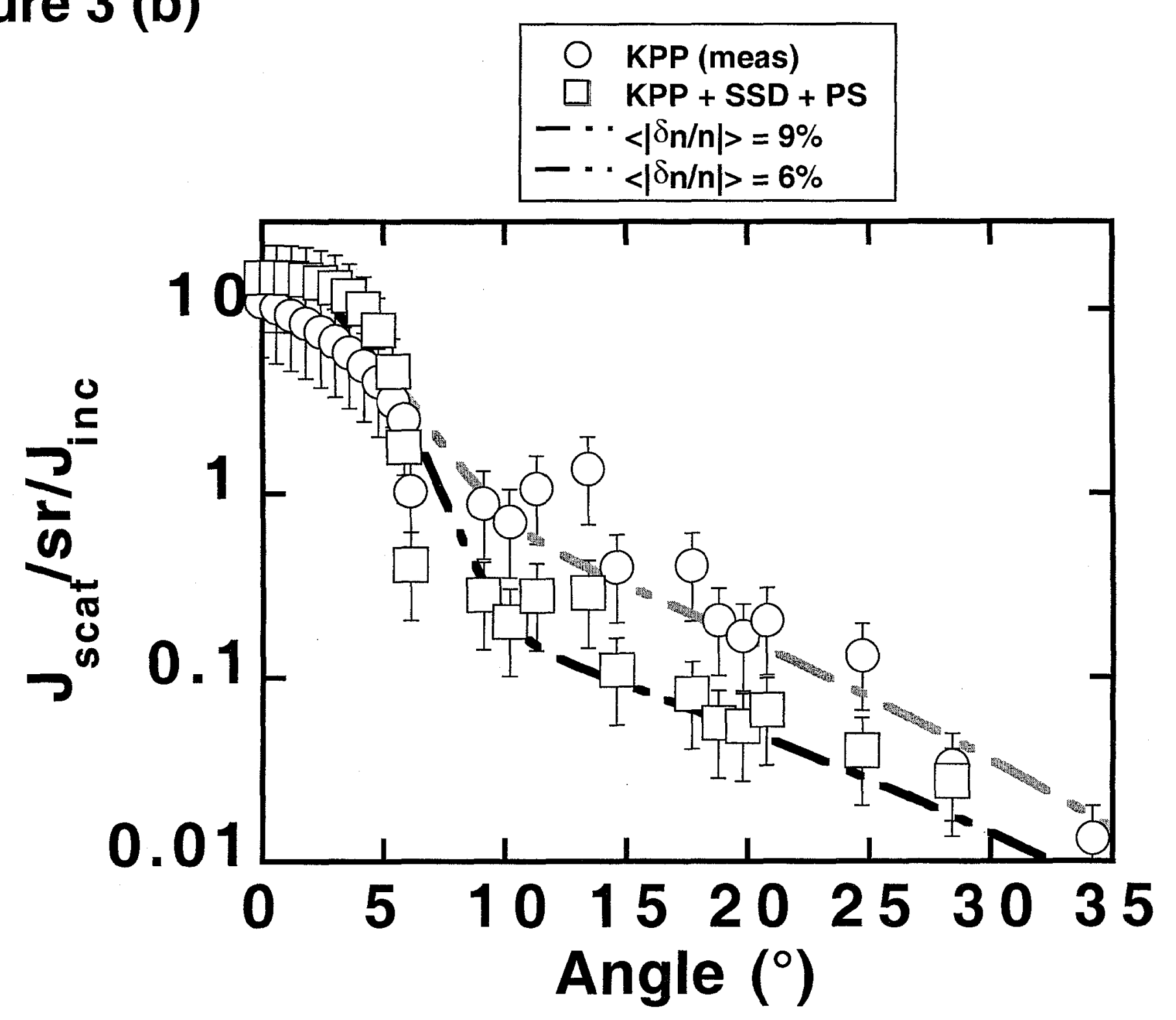


Figure 4

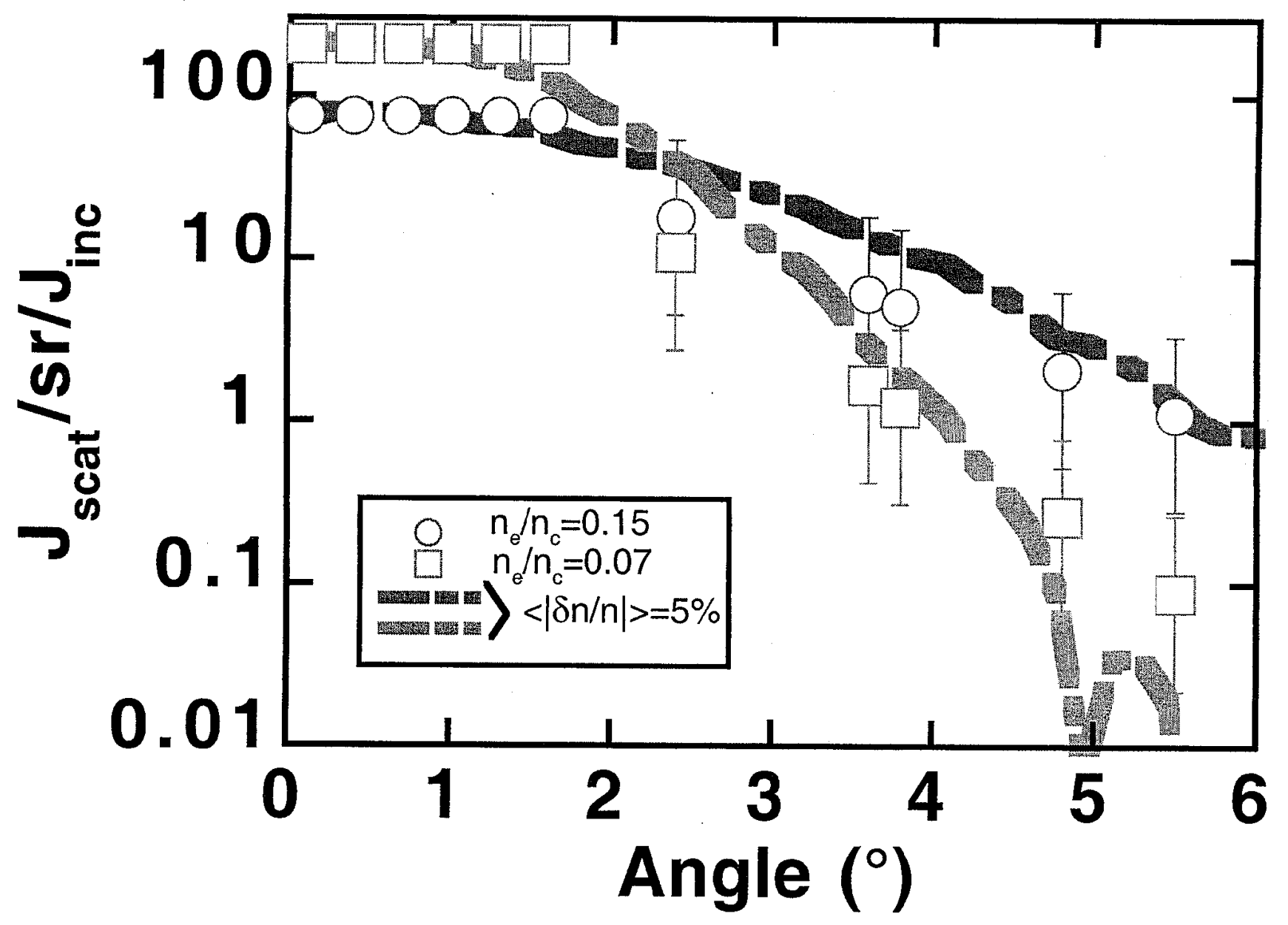




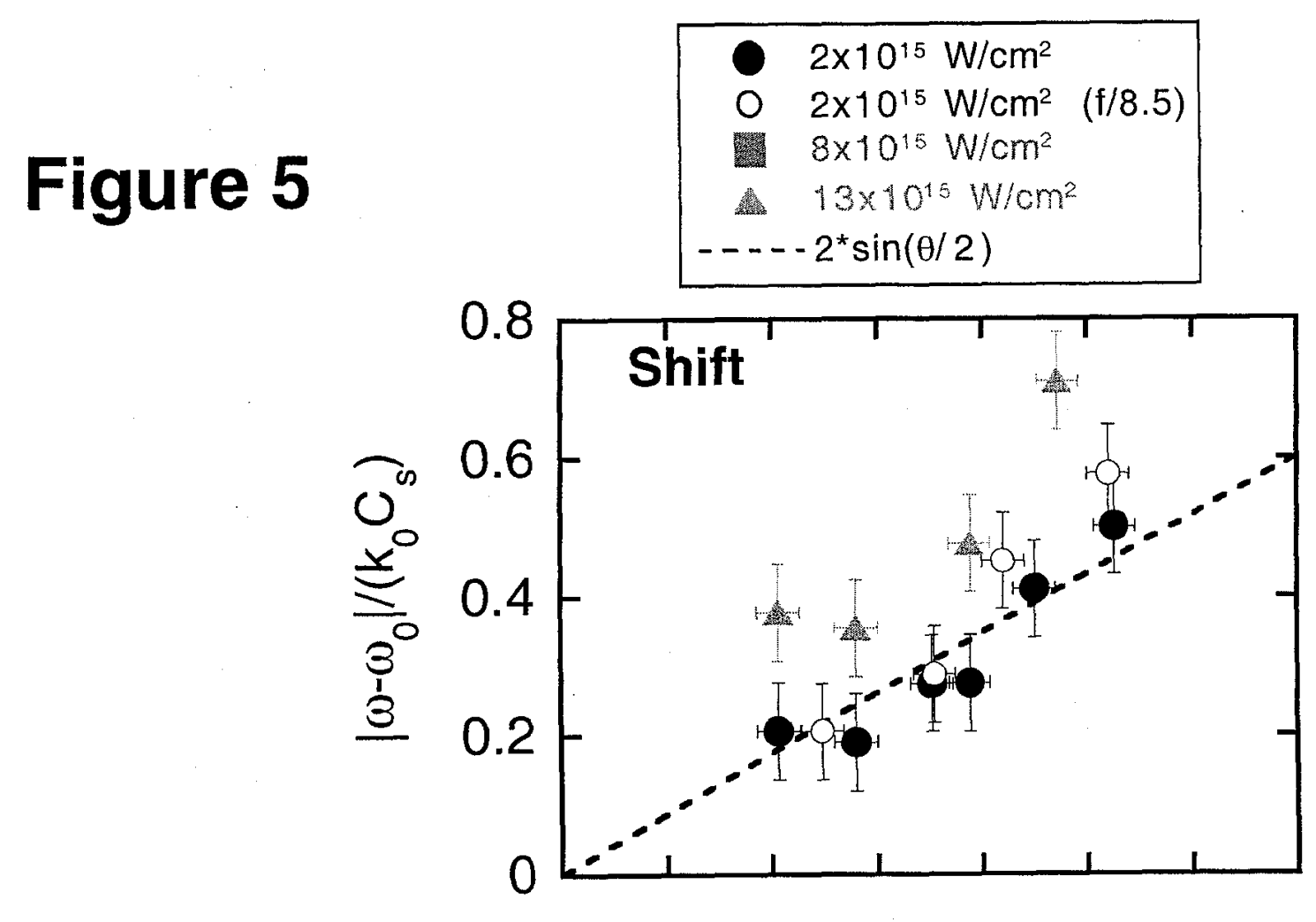

(a)

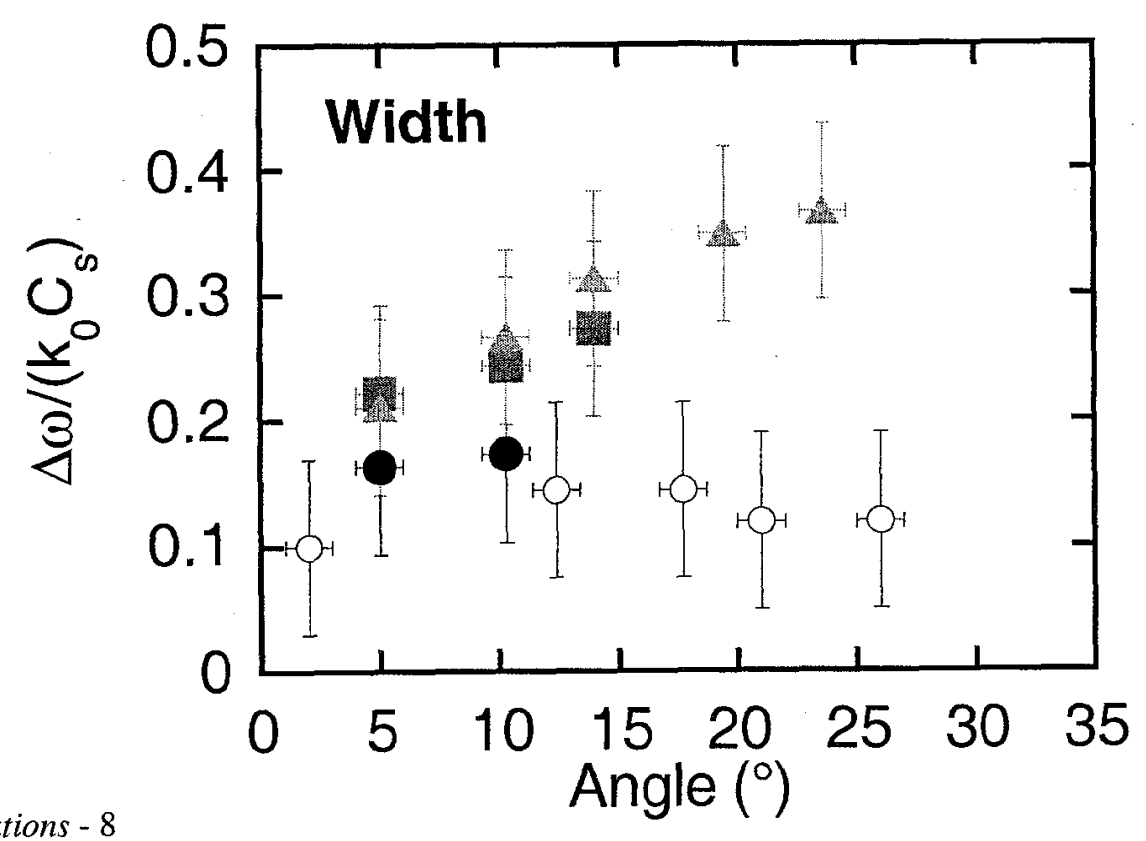

(b) 
Figure 6

(a)

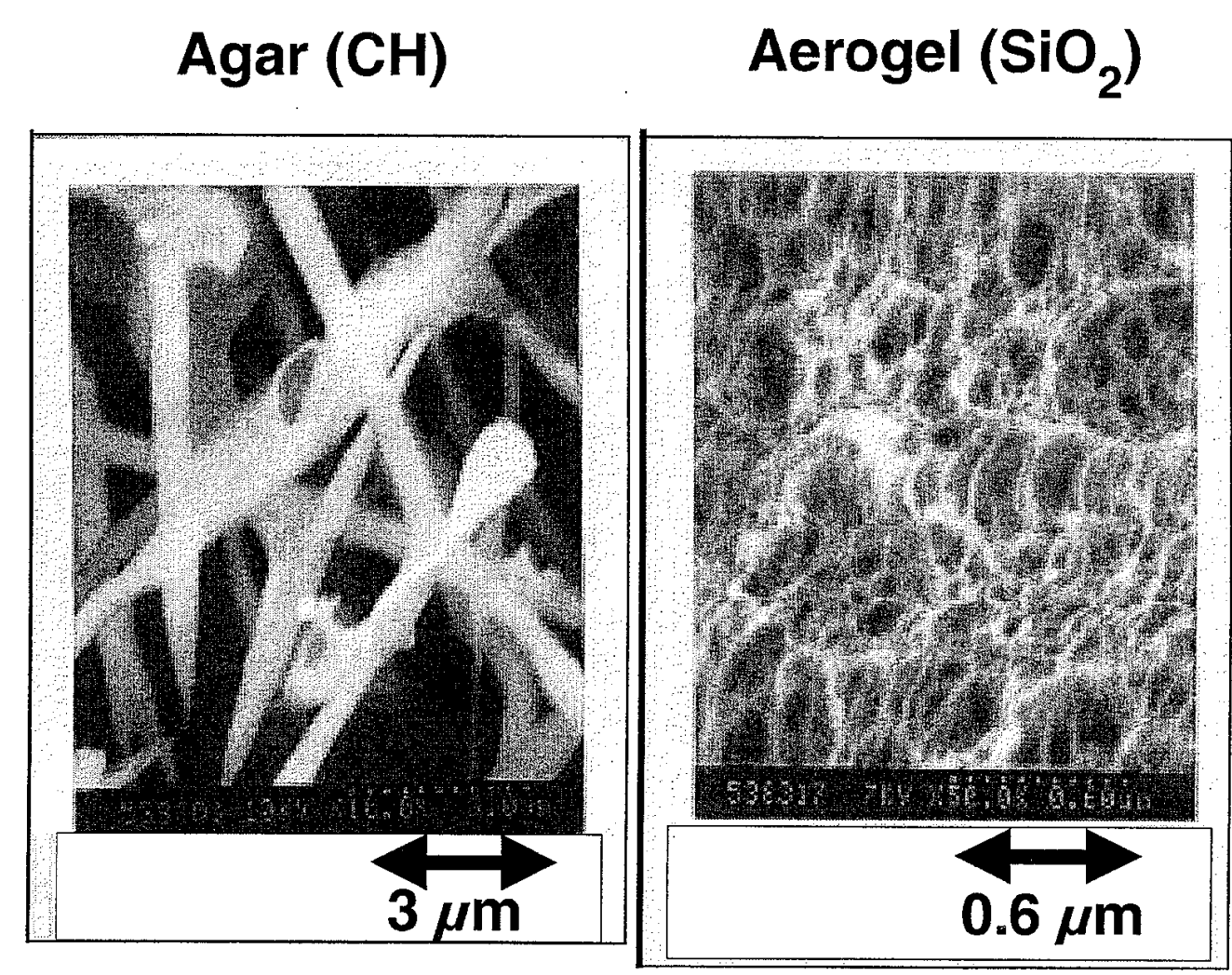

(b) Aerogel $\left(\mathrm{SiO}_{2}\right)$ 
Figure 7

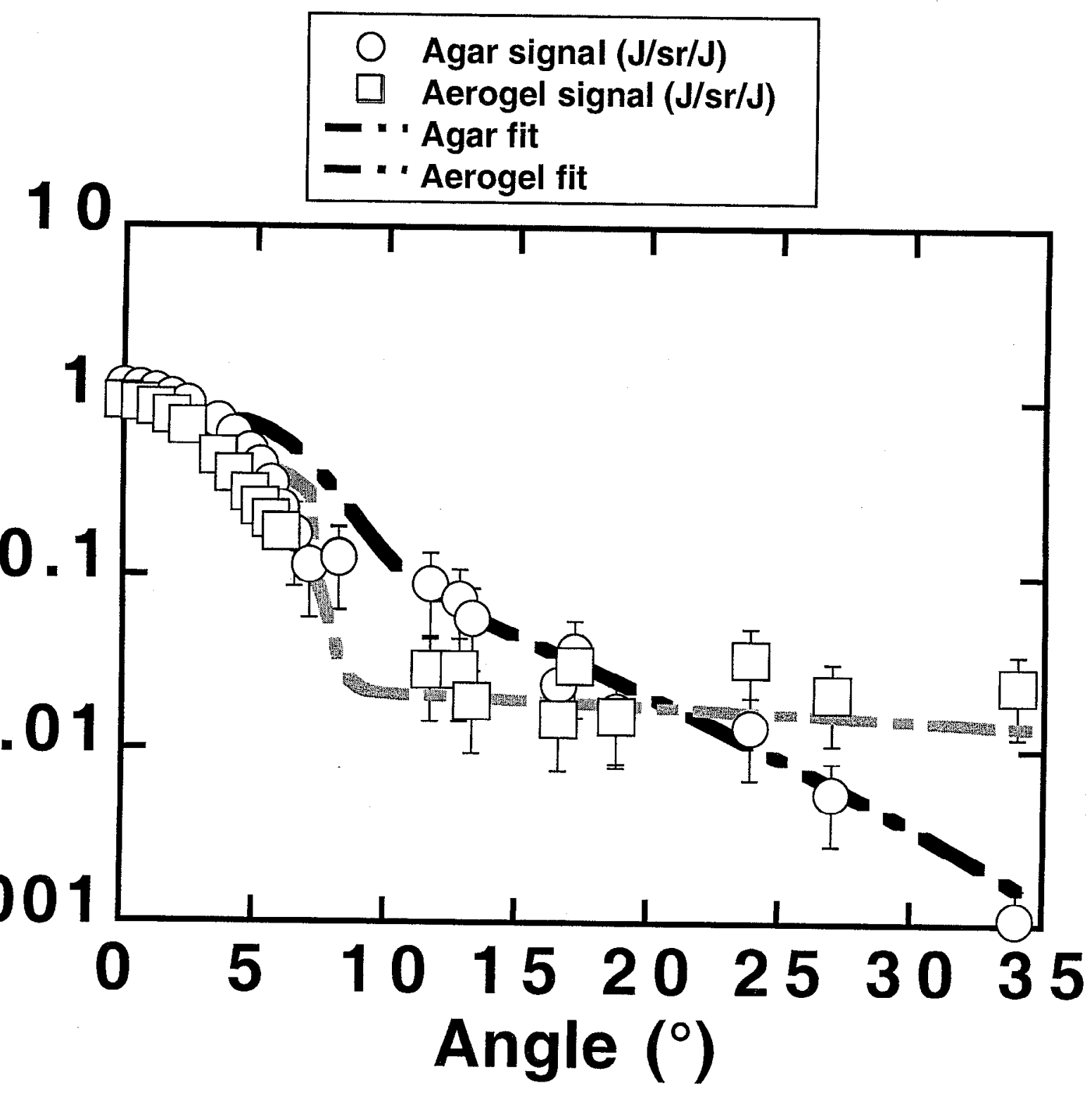




\section{Figure 8 (a)}

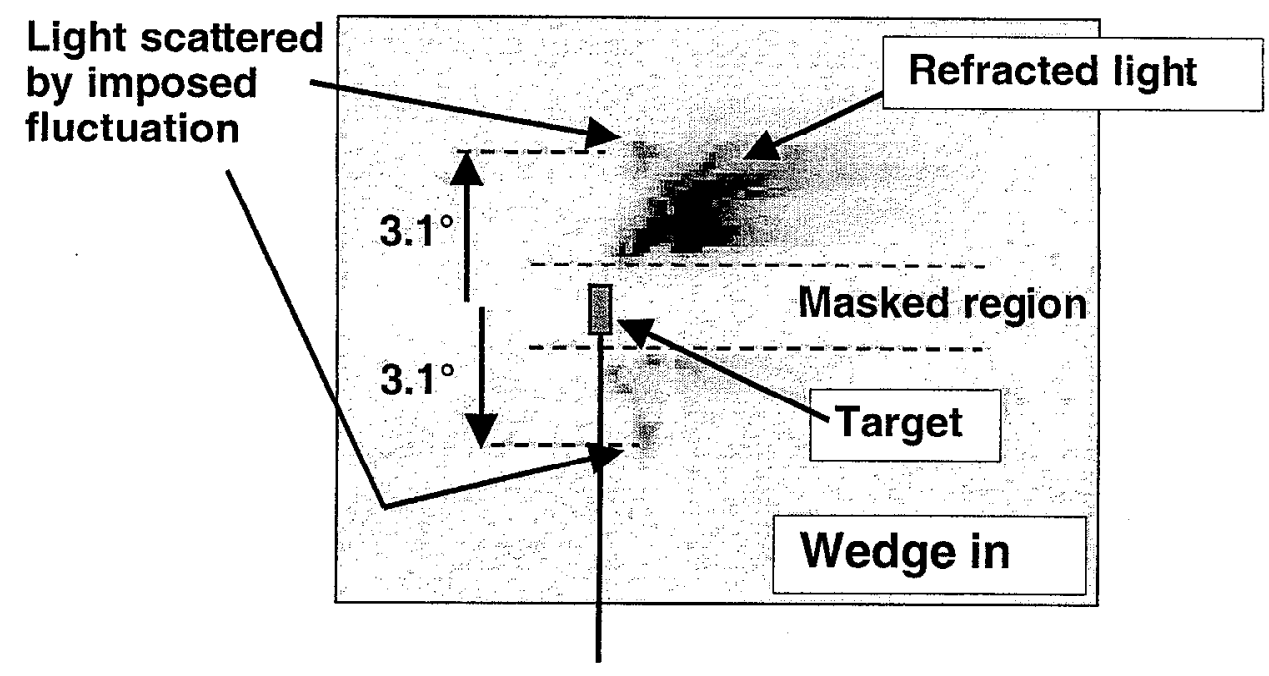

\section{Figure 8 (b)}

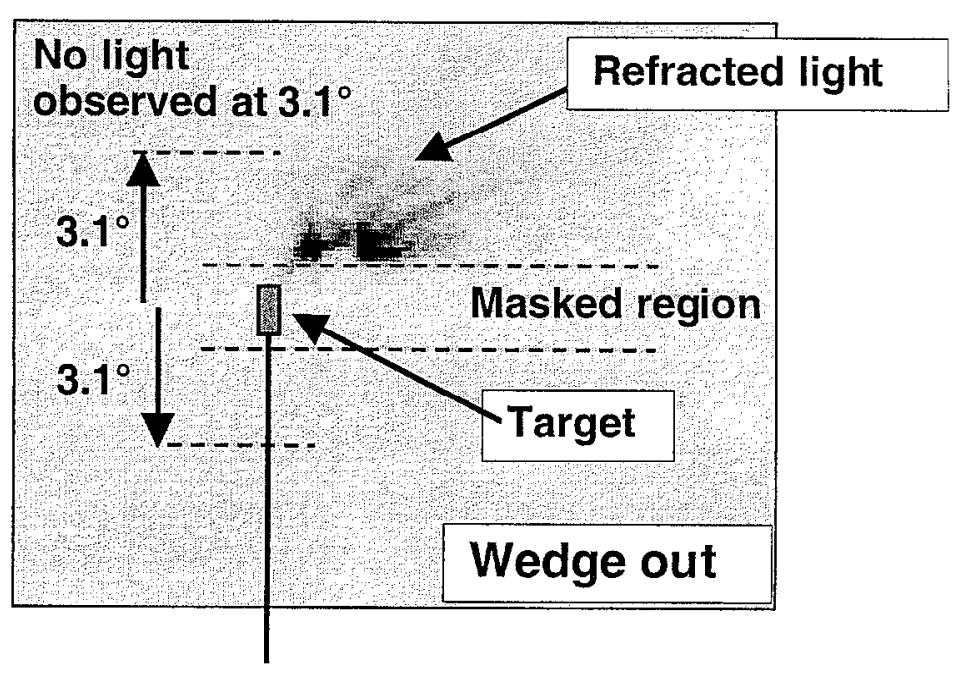




\section{Figure 9}
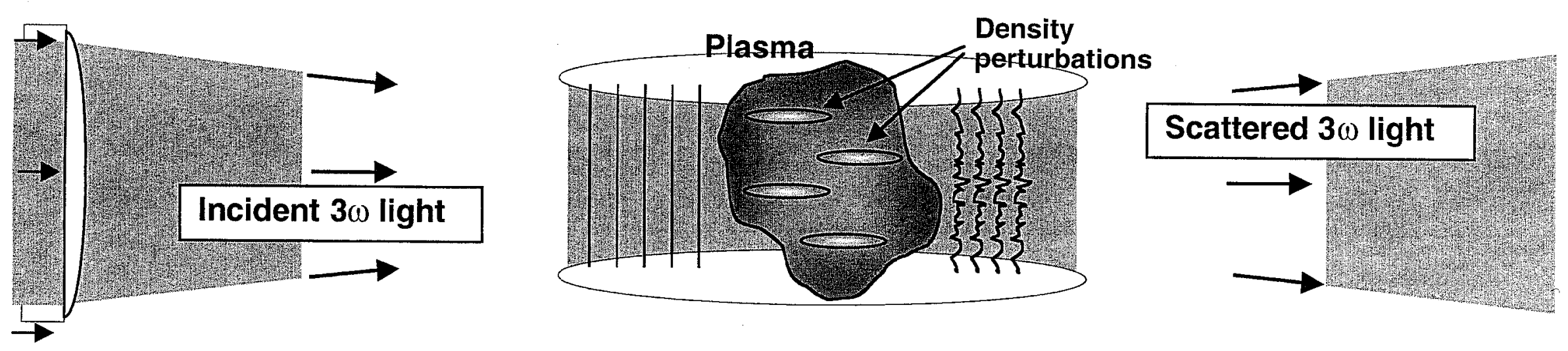
Figure 10 (a)

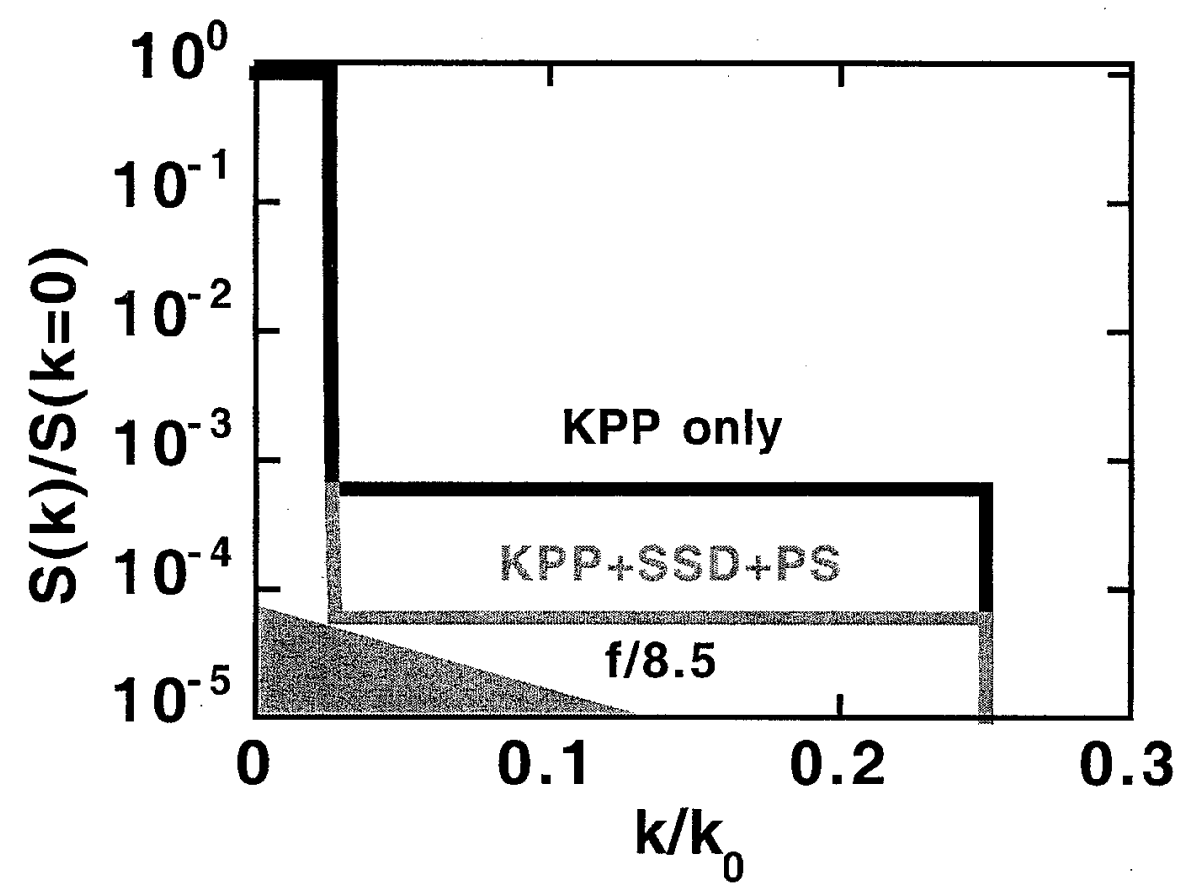

Figure 10 (b)

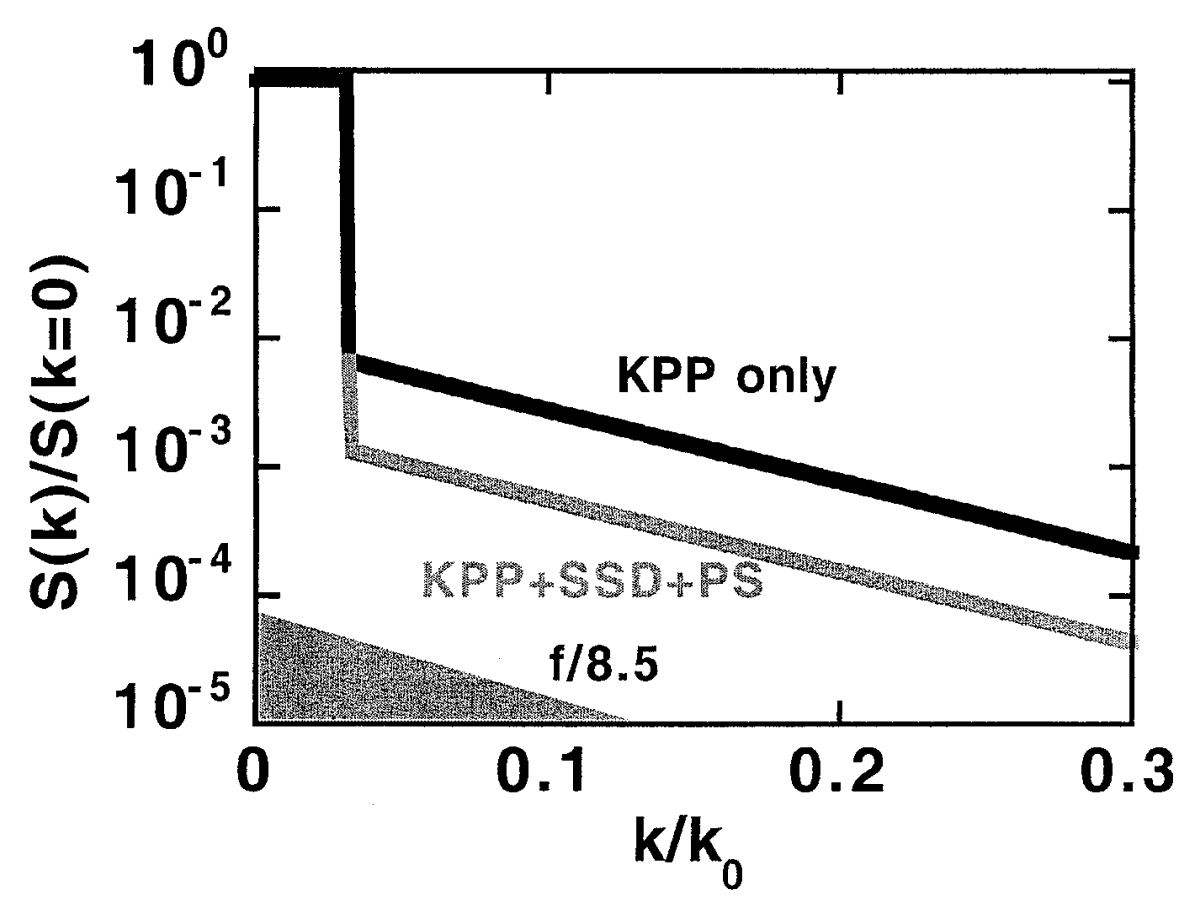


Figure 11

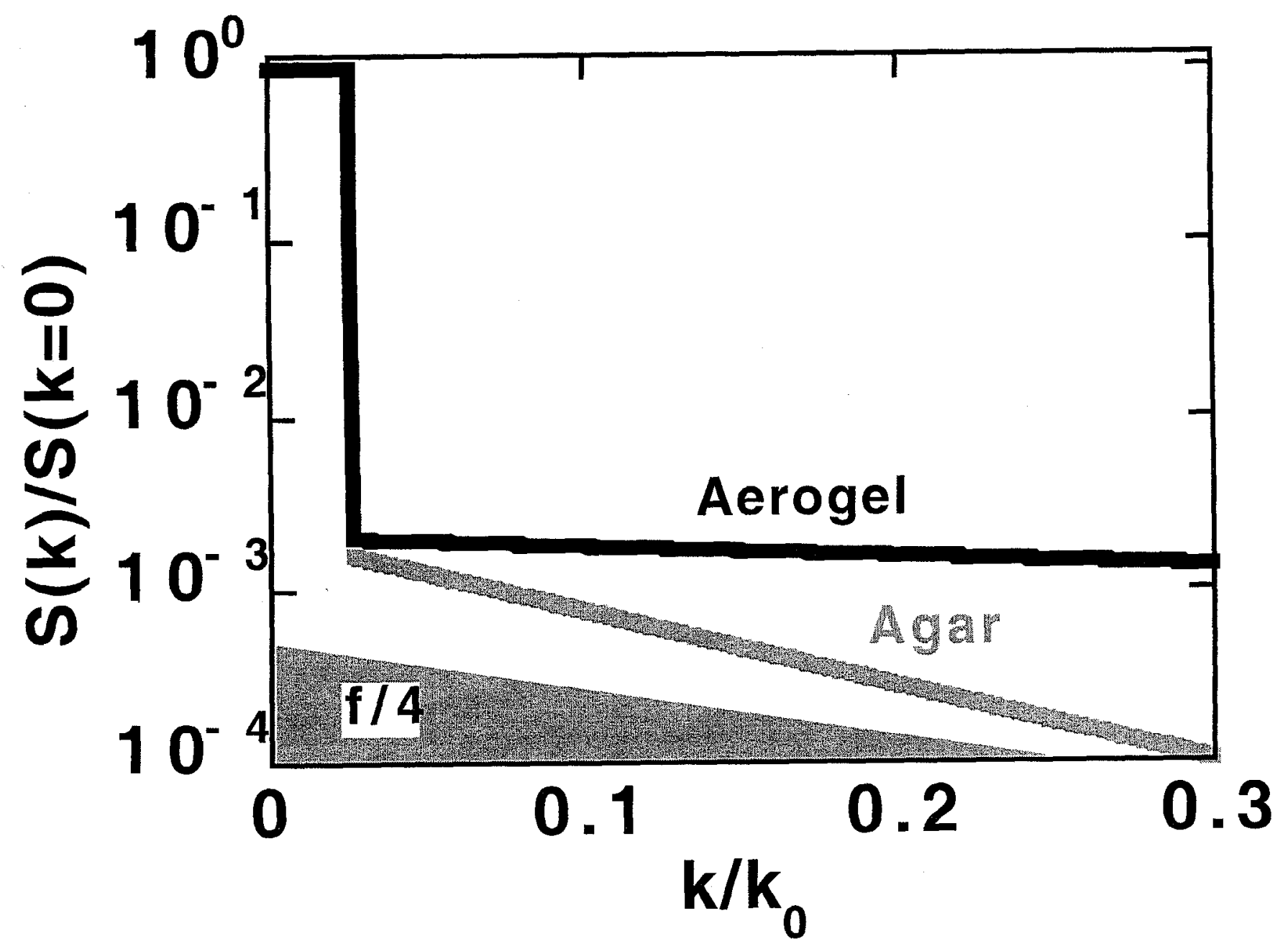


Figure 12 (a)

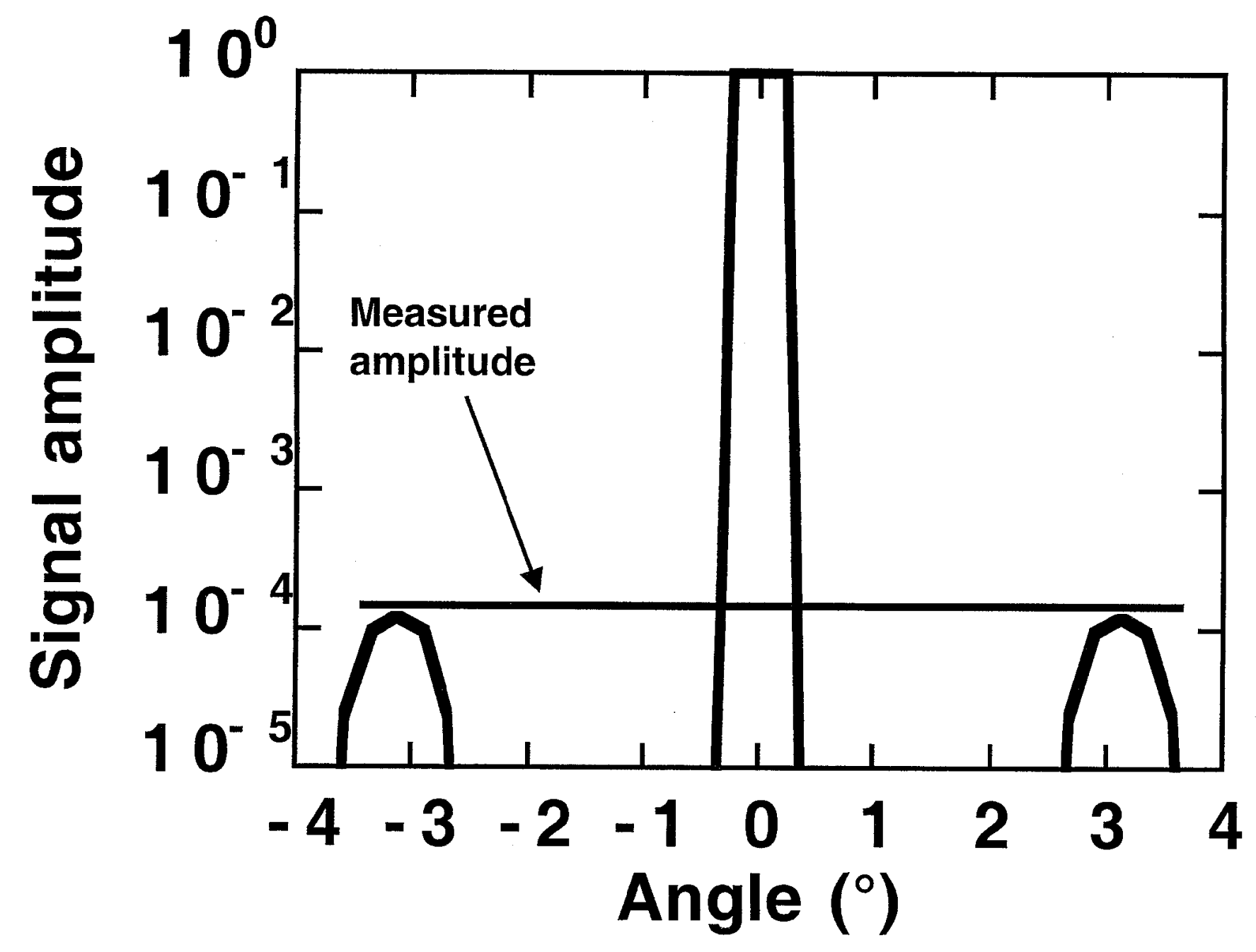


Figure 12 (b)

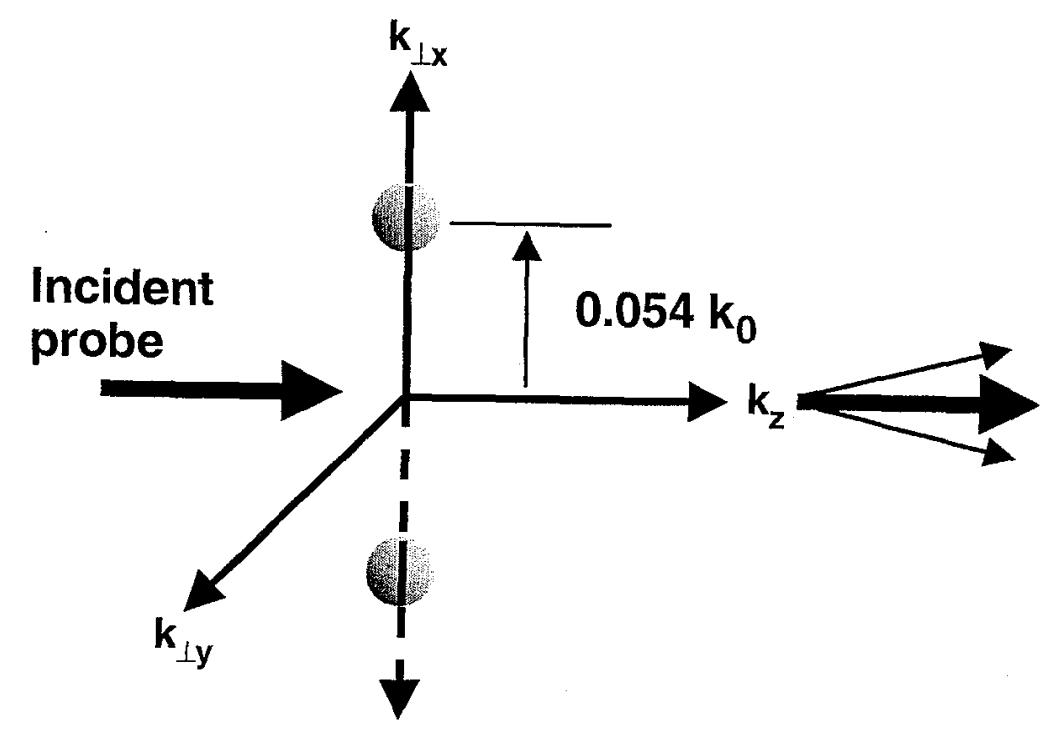


